\title{
Avoiding The Man on the Wire: Improving Tor's Security with Trust-Aware Path Selection
}

\author{
Aaron Johnson*, Rob Jansen*, Aaron D. Jaggard*, Joan Feigenbaum ${ }^{\dagger}$ and Paul Syverson* \\ *U.S. Naval Research Laboratory, \{aaron.m.johnson, rob.g.jansen, aaron.jaggard, paul.syverson\}@nrl.navy.mil \\ †Yale University, joan.feigenbaum@yale.edu
}

\begin{abstract}
Tor users are vulnerable to deanonymization by an adversary that can observe some Tor relays or some parts of the network. We demonstrate that previous network-aware path-selection algorithms that propose to solve this problem are vulnerable to attacks across multiple Tor connections. We suggest that users use trust to choose the paths through Tor that are less likely to be observed, where trust is flexibly modeled as a probability distribution on the location of the user's adversaries, and we present the Trust-Aware Path Selection algorithm for Tor that helps users avoid traffic-analysis attacks while still choosing paths that could have been selected by many other users. We evaluate this algorithm in two settings using a high-level map of Internet routing: $(i)$ users try to avoid a single global adversary that has an independent chance to control each Autonomous System organization, Internet Exchange Point organization, and Tor relay family, and (ii) users try to avoid deanonymization by any single country. We also examine the performance of TrustAware Path selection using the Shadow network simulator.
\end{abstract}

\section{INTRODUCTION}

Tor is a popular tool for low-latency anonymous communication, with over an estimated 1.5 million daily users. In order to use Tor to communicate with others, clients choose a three-hop path from the set of over 7000 relays volunteering bandwidth to the network. In order to balance load among the relays, and in particular to optimize the allocation of Tor's limited bandwidth, the default path selection algorithm is bandwidth-weighted so that a client will select a relay with a probability equivalent to the ratio of that relay's available bandwidth to the total network bandwidth capacity. Clients communicate with arbitrary Internet hosts via a cryptographic circuit with a layer of encryption for each of the three relays on its path, which are termed the entry guard, middle, and exit, according to their position on the path. Because this circuit is built using a telescoping process, it provides unlinkability against a passive, non-global adversary that cannot observe both ends of the circuit.

Unfortunately for many Tor users, a global adversary that can observe both ends has become a very real and significant threat. An adversary in such a position can perform a "firstlast" traffic-correlation attack for any of the circuit's streams (i.e., TCP connections to destinations multiplexed over circuits) by using similarities in the volume and timing of the traffic at both ends to match them with each other, thereby deanonymizing the user. These techniques are efficient and effective [28]. In order to carry out traffic-correlation attacks, an adversary must be in a position to $(i)$ observe Tor traffic on an Internet path between a client and its chosen entry guard or control that entry guard, and (ii) observe an Internet path between the selected exit relay and the destination or control the exit or destination. Due to Tor's volunteer-relay model and its bandwidth-weighted path-selection algorithm, an adversary may get in a position to observe a large amount of traffic simply by running a fast relay, and it can otherwise observe traffic by controlling or coercing entities on the paths to and from relays including Internet Service Providers (ISPs), Autonomous Systems (ASes), and Internet Exchange Points (IXPs).

Previous approaches to improving resilience against traffic observation and correlation attacks have been limited in nature and only consider specific threats. One main approach focuses on defeating an adversary that observes an AS or IXP [7], [9], [13], [15], [24], [29], [30] and suggests creating Tor circuits such that the set of ASes and IXPs that appear on the Internet paths between the client and guard is disjoint from the set of ASes and IXPs between the exit and destination. However, this solution ignores the critical effects of multiple connections over Tor, which under this approach leak increasing amounts of information that can allow the adversary to determine a client's location. We present attacks of this nature on Astoria [30], a recent proposal of this sort, with our results showing that even a moderately-resourced adversary can identify the client's AS within seconds. These attacks have similar implications for all path-selection proposals using this approach.

The other main approach focuses on an adversary that can observe some Tor relays [21], [22] and suggests that, for the most sensitive circuit positions, users choose a small number of relays from among those the user trusts the most. However, this approach leaks information to an adversary that can eventually identify the trusted relays (e.g., via a congestion attack [14], [16]) and uses a restrictive trust model. No existing solution to traffic correlation attacks provides protection from the variety of attacker resources and tactics that recent research and experience suggests is realistic [23].

In contrast, this paper develops defenses against traffic correlation that are based on a general probabilistic model of network adversaries. Using this model we can consider adversaries with diverse resources, such as those that observe network traffic at any combination of network providers, exchange points, physical cables (undersea or elsewhere), and Tor relays. The model allows us to incorporate uncertainty about and randomness in an adversary's actions. It also enables us to express common factors underlying the adversaries' ability to compromise different network locations, such as shared legal jurisdiction or corporate ownership. A user expresses a trust belief about her adversaries by specifying a probability distribution over their presence at a set of network locations, and she turns this belief into a trust policy by including a weight for each adversary indicating her relative level of concern about that adversary. 
Using these trust policies, we design Trust-Aware Path Selection (TAPS), a novel path-selection algorithm that uses trust in network elements to inform a user's decision about how to select relays for its Tor path. Using TAPS, clients select paths so as to minimize the probability that an adversary is in a position to observe both ends of their Tor circuit while at the same time ensuring that their path selection behavior does not harm their security by making them stand out from other clients. In addition to defending against a much broader class of adversaries, TAPS addresses the other deficiencies of prior proposals. In particular, it provides security against an adversary that can monitor and link user activity across multiple connections, influence how users make connections, and identify the relays used repeatedly by a client.

In order to facilitate the adoption of TAPS, we describe both a long-term and a short-term deployment strategy. In the long-term strategy, all Tor users participate and use the TrustAll version of TAPS to replace Tor's existing bandwidthweighted algorithm. In the short-term strategy, TAPS provides the option for security-conscious users to use trust to defend against traffic-correlation attacks while most users continue to use "vanilla" Tor (i.e., bandwidth-weighted path selection). We design the TrustOne TAPS version for this case, in which users must both avoid traffic correlation and choose paths that blend in with the vanilla Tor users.

We evaluate the security of TAPS via simulation with modified versions of the Tor Path Simulator (TorPS) [23]. This evaluation is done with respect to two plausible and illustrative trust policies: (i) The Man policy, in which a single adversary has an independent probability of compromising each $A S$ organization (i.e., group of ASes run by the same entity), IXP organization, and self-declared relay family (i.e., set of relays run by the same entity) in Tor; and (ii) the Countries policy, in which each country is considered a potential adversary and observes all ASes, IXPs, and relays located inside of it. Our analysis of The Man policy for a popular Tor client location shows a reduction in the probability of a successful first-last attack from about 0.7 to about 0.4 in TrustAll with typical webbrowsing activity over the first week in December 2013, and from about 0.68 to as little as 0.1 in TrustOne with repeated connections over the same week to a single IRC server popular with Tor developers. Our analysis of the Countries policy over that week shows a reduction in the median number of countries that "unnecessarily" compromise a stream (i.e., compromise a stream when they don't contain both the client and destination) from 5 to 2 in TrustAll with typical user behavior.

Our algorithms are designed not only to improve security, but also to allow security to be traded off for performance. They achieve this by allowing clients to configure the fraction of bandwidth weight that their trusted sets of candidate guards and exits should exceed before making a bandwidth-weighted choice from them. This mechanism results in a client selecting from among the most secure relays while still making use of much of the network and doing so in a bandwidth-weighted manner. We explore these trade-offs using the Shadow simulator [3], [19] and find that there exist parameters that result in only a slight decrease in performance, and only for less than 5 percent of users.

This is the full version of the conference paper [20]. The additions in this version include a discussion of repeatedconnection attacks and a measurement-based study of the risk of cross-circuit attacks.

\section{Attacks on Network-Aware Path Selection}

There have been several proposals to improve Tor security by considering the network entities (e.g., AS or IXP) that can observe a circuit [7], [9], [13], [15], [24], [30]. However, none of these works considers anonymity across multiple Tor connections. Any realistic use of Tor involves multiple connections from the same user, though, and these connections are linkable by the adversary in many important cases, which means that it doesn't suffice to consider the security of an individual connection. Indeed, we present two specific attacks that can deanonymize users by identifying and analyzing multiple connections from the same user.

While there have been several proposals for networkaware path selection, we focus on just one for concreteness: Astoria [30]. It is among the most recent and complete proposals, and it provides a valuable example as many of the others suffer the same weaknesses. Astoria is designed to prevent deanonymization by an AS (or group of sibling ASes controlled by the same organization). To choose a circuit for a given destination, a client determines the ASes between the client and its guards and between the exits and the destination. It then makes a bandwidth-weighted random choice from among the guard-exit pairs such that no AS appears on both the client side and the destination side. If no such pair exists, a linear program is used to determine the selection distribution over guard-exit pairs that minimizes the maximum probability that some AS appears on both sides. Circuits are reused for destinations within the same AS.

Chosen-Destination Attack: Consider an adversary that runs a number of web servers in different locations and runs some Tor relays. If a Tor user visits one of the malicious web servers, the adversary can force the browser to visit the other malicious servers and request resources that are linkable to the original request (e.g., images with unique names). The adversary will then observe the pattern of exits chosen by the client to visit the servers in different locations, and it will also observe some of the client's guards if the malicious relays are ever selected in the middle position. This attack strategy applies more generally to any situation in which the adversary can choose the destinations that the client visits and is able to link those connections as originating at the same client.

Under this attack, path-selection algorithms that choose relays based on the client's location can leak increasing amounts of information about that location with each additional destination visited. This is the case for Astoria, and we demonstrate that this attack is effective on that system.

To demonstrate the attack, we construct an AS-level Internet map using traceroute-based topology data and inferred AS relationships from CAIDA [11] and BGP routing tables supplied by Route Views [35]. We use the data from these sources for October 2015. Routes between AS locations on our map are inferred using the algorithm proposed by Qiu and Gao [33], which takes AS paths observed in BGP tables and extends them to other locations using shortest "valley-free" paths. Conversion of IPs to ASes is performed using routing prefix tables from Route Views [34].

We also use data from Tor Metrics [5] to evaluate the attack's effectiveness as if it were run on the Tor network in the recent past. We use the archived network consensuses and server descriptors to determine such facts as which relays existed, what families they were grouped in, what their relative 
bandwidth capacities were, and what their "exit policies" were. We use the first network consensus from October 2015 and its listed descriptors. We also use data from Juen [24] that identifies 414 Tor client ASes as ever being observed making a Tor connection and shows their relative popularity. 389 of these appear in our AS map and are used in our analysis. The five most popular client ASes identified by Juen, in order from most popular, are 6128 (Cable Vision Systems, US), 25019 (SaudiNet, SA), 8972 (PlusServer AG, DE), 6893 (Saitis Network, CH), and 15467 (Enternet Libercom, HU).

We simulate Astoria's path selections for 1000 destinations in ASes selected uniformly at random from those 44626 ASes in a maximal fully-connected component in our map that also advertise prefixes. For each destination AS, an IP is chosen arbitrarily from an advertised prefix, and a connection to port 443 is simulated. We repeat the simulation 100 times. We suppose that the adversary runs the 4 relays with the largest probabilities of being selected as a middle, which have a total advertised bandwidth of $1.01 \mathrm{Gbps}$ and a cumulative probability of $3.3 \%$ of being selected as a middle.

Table I shows how often the adversary observes some or all of the client's guards when the client uses 3 guards. It shows that with 100 destinations the adversary observes all guards with $30 \%$ probability, and with 300 destinations it observes all guards with $94 \%$ probability. It sometimes observes guards even with no destinations due to being selected as a guard.

TABLE I: Probability of observing the client's guards

\begin{tabular}{|l|l|l|l|l|}
\cline { 2 - 5 } \multicolumn{1}{c|}{} & $\begin{array}{l}\text { Pr. 0 guards } \\
\text { observed }\end{array}$ & $\begin{array}{l}\text { Pr. 1 guard } \\
\text { observed }\end{array}$ & $\begin{array}{l}\text { Pr. 2 guards } \\
\text { observed }\end{array}$ & $\begin{array}{l}\text { Pr. 3 guards } \\
\text { observed }\end{array}$ \\
\hline 0 destinations & 0.96 & 0.04 & 0 & 0 \\
\hline 100 destinations & 0.04 & 0.14 & 0.52 & 0.30 \\
\hline 200 destinations & 0 & 0.01 & 0.25 & 0.74 \\
\hline 300 destinations & 0 & 0 & 0.06 & 0.94 \\
\hline
\end{tabular}

We then consider how well the adversary can guess the client's AS after the attack in the case that he observes all of the client's guards. We again simulate Astoria's path selections for 1000 random destination ASes and repeat the simulation 100 times. We follow Nithyanand et al. [30] in using 3 guards (1 and 2 guards yield similar results). We suppose that the adversary uses a uniform prior distribution on the 389 client ASes. We then compute the adversary's conditional distribution on the client ASes given the observed guard set and the sequence of exits. The average entropy of the resulting distributions as we increase the number of attack destinations is shown for the top 5 client ASes in Figure 1. It shows an expected steady decrease in entropy for all client locations as the attacker uses more destinations. By 300 destinations, all locations result in less than 4 bits of entropy on average, and by 1000 destinations, the average entropy is less than 2.5 bits for all locations. Identifying the client AS could be very dangerous for a Tor user, as it can identify the country of that user as well as the ISP whose network logs could be used to completely identify the user. Note that this attack could be completed within the time needed to construct circuits and open streams on them in parallel, which is on the order of seconds.

Repeated Connections: Consider a malicious destination to which a user repeatedly connects over time. A new circuit is created for each connection that is separated by enough time and inactivity (Tor uses a new circuit after 10 minutes by default, although Tor Browser extends this for sufficiently-

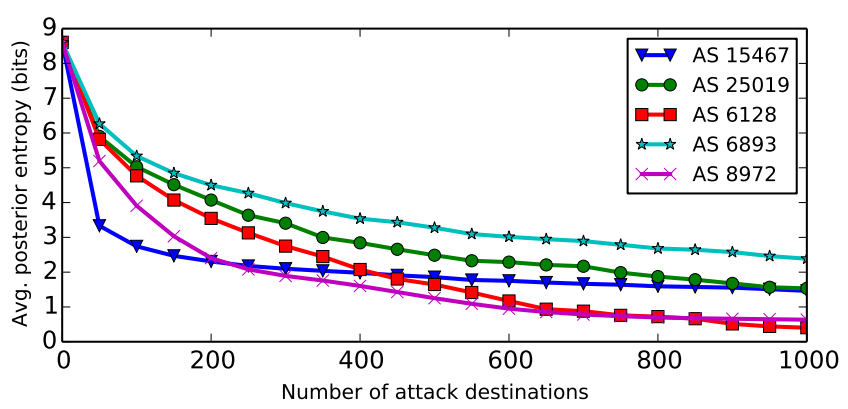

Fig. 1: Avg. entropy of posterior client-AS distribution after chosendestination attack on Astoria

frequent connections). Suppose that the adversary can link those connections as originating at the same user (e.g., by pseudonymous login at the server). If the adversary also runs a relay, it can eventually learn both the client's guard set (by being used occasionally as the middle relay) and the exit distribution used to reach the destination from each guard (by observations at the destination). If these distributions depend on the user's location, as in Astoria, the adversary can thus narrow down the user's location over time.

Cross-Circuit Attack: Consider a popular website that causes the user's browser to fetch resources of (roughly) known sizes from a set of servers in other ASes. An AS-avoiding path-selection algorithm like Astoria may create new circuits for these new destinations that place a malicious AS between an exit and server on one connection and between the client and a guard on another connection. If the adversary can link its observations on those two connections, which it might by using the timing and amount of traffic on the circuits, then it can deanonymize the user, effectively performing a crosscircuit correlation attack. Linking observations across circuits may be especially easy when the adversary observes the first connection to a website from an exit because then it can compare traffic on subsequent connections to a traffic fingerprint that it has previously measured for that website that contains the timing, amount, and destinations of traffic on connections initiated by that website. In contrast to the chosen-destination and repeated-connections attacks, this attack can be performed when the destinations are honest but some AS is malicious. This kind of attack enables complete deanonymization by an AS via timing correlation even when each individual circuit is not vulnerable to a correlation attack. Figure 2 illustrates an example of the cross-circuit attack.

We evaluate the potential vulnerability of Astoria to such an attack by examining how it constructs and uses circuits when visiting popular websites. To measure the connections made during such a visit, we use Tor Browser to fetch the front page from each of the Alexa global top 5000 websites [1]. This process is automated using tor-browser-selenium [39]. We disable automatic updating and browser caching, and we reload Tor Browser between each website visit. These steps help ensure that we only consider streams initiated by the website visit and that we observe all streams normally initiated by a fresh visit. We record all of the streams constructed during each visit using Tor's control port [38]. For each visit, we extract a list of the IP addresses and TCP ports to which streams are opened, excluding any streams generated by automated Tor 


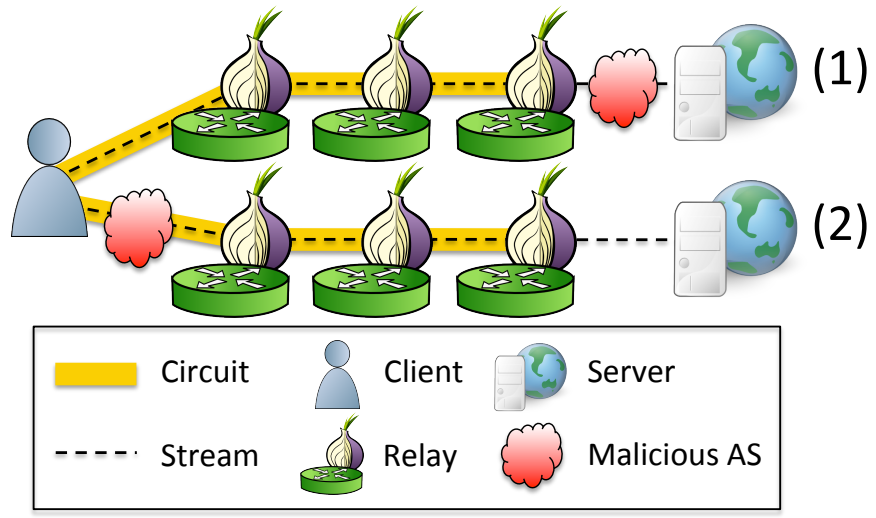

Fig. 2: Example of a cross-circuit attack: a malicious AS observes the first connection made to a server during a website visit (1), then observes the client's connection to a guard on a subsequent connection during the same website visit (2), and then can conclude that the observed client visited the observed website.

events such as consensus updates. We remove from these lists those IP addresses for which a mapping to AS number doesn't exist, again using the Route Views data [34] to determine the mapping. We also manually inspect the returned page contents to verify that the website loaded successfully.

We used Tor Browser v.6.0.4 to perform a crawl of the top 5000 sites on September 1st, 2016. Of these, 2573 visits reached the desired site and resulted in a non-empty list of IP addresses and ports. Reasons for failure include network error, interception by CloudFlare, a redirect loop, timeout (set at 45s), and missing IP-to-AS mappings. Among these 2573 websites, the number of unique pairs of IP address and TCP port connected to during the visit ranges from a minimum of 1 to a maximum of 160 with a median number of 17. This suggests that there are many popular websites for which Astoria may construct many new circuits and thus be vulnerable to a cross-circuit attack.

To evaluate this, we simulate circuits in Astoria when visiting each website much as we simulated Astoria when evaluating the chosen-destination attack. We again determine AS-level paths using Qiu-Gao inference on our AS map, we use the same 389 popular client locations, and we use the same data from Tor Metrics to model the relays in the Tor network. Then, for each of the 2573 websites reached successfully and for each client location, we simulate 100 times how Astoria would create and use circuits to reach the sequence of addresses and ports that were observed for the given website. We simulate Astoria with 3 guards, as this is the recommended configuration. Fewer guards would reduce the possibility of a cross-circuit attack (it would be eliminated with 1 guard) but would only reduce Astoria's protection against the standard "direct" correlation attack that it is designed to mitigate.

The resulting number of circuits constructed varied significantly depending on the website. If we aggregate the simulations by website and consider, for each website, the median number of circuits constructed over all simulated visits to that website, we see over all websites a minimum of 1 circuit, a median of 8 , and a maximum of 54 . If we instead aggregate the simulations by client location and consider, for each client location, the median number of circuits constructed over all simulated visits by that client location, that value is 8 circuits for every client location. Thus the number of circuits constructed is highly dependent on the destination. This result is unsurprising, as new circuits are constructed either when an IP address in a new AS is encountered or when a new TCP port is incompatible with the existing exit's policy, both of which are heavily dependent on the website. These results about circuit construction also show that, for many popular websites, Astoria frequently provides many new circuit creations and thus opportunities for a cross-circuit attack.

We evaluate the vulnerability of each simulated website visit to a direct correlation attack and to a cross-circuit correlation attack. A visit is considered vulnerable to a direct attack if there exists some AS $A$ such that, for some stream, $A$ appears both between the exit relay and the destination host and between the client and guard. For this evaluation, we consider an AS to be between two Internet locations $B$ and $C$ if it is present on either the AS path from $B$ to $C$ or the AS path from $C$ to $B$ (note that both $B$ and $C$ are thus considered between $B$ and $C$ ). A website visit is considered vulnerable to a cross-circuit attack if there exists an AS $A$ such that $(i) A$ is between the exit and the destination host on the first stream, (ii) $A$ is between the client and the guard on some stream, and (iii) $A$ is not in a position to perform a direct attack during the visit as defined previously.

Figure 3 shows how often Astoria is vulnerable to these attacks as a distribution over all pairs of the 389 client locations and 2573 websites. It shows that, while Astoria does effectively

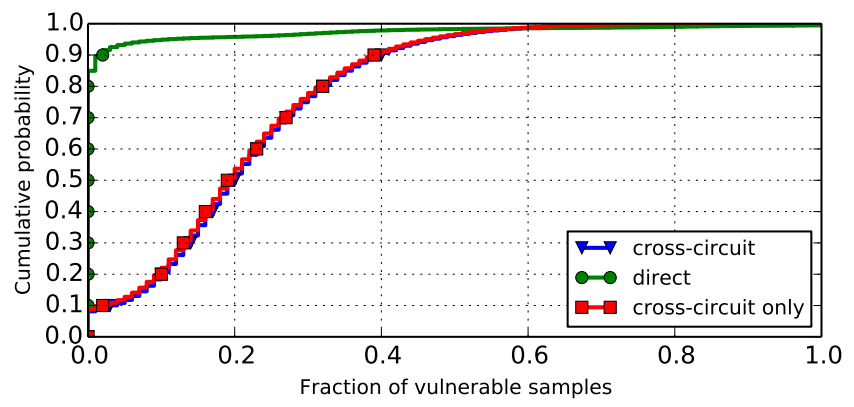

Fig. 3: CDF over pairs of client location and website of the fraction of simulated visits under Astoria that are vulnerable to a cross-circuit attack, a direct attack, and a cross-circuit attack but no direct attack.

prevent most direct correlation attacks, for many popular client locations and websites, users are frequently vulnerable to deanonymization via a cross-circuit attack. Indeed, while the median fraction of simulated visits vulnerable to a direct attack is only 0.03 , the median fraction vulnerable to a cross-circuit attack is 0.2 . To more clearly consider the risk added by the cross-circuit attack to the risk of direct attack, we show the frequency distribution of visits that are vulnerable to a crosscircuit attack but not a direct attack. The median frequency of this cross-circuit-only vulnerability is 0.19 , which shows that the cross-circuit attack is a major added risk.

These results expose a fundamental problem with Astoria's strategy of varying the guard depending on the destination. Choosing two different guards for two different destinations is useful when for both guards some AS between the client and guard appears between the exits and destination for one destination but not the other, which is precisely the situation when some AS is in a position to perform the cross-circuit at- 
tack. This suggests that, unless the client can somehow identify which pairs of connections are vulnerable to being linked as originating at the same client, it should use few guards and choose them for circuits oblivious to the destination.

\section{TRUST MODEL}

\section{A. Trust Policies}

We use the definition of network trust given by Jaggard et al. [17]. A trust belief is a probability distribution that indicates how likely adversaries are to observe traffic at different network locations. A location is considered to be more trusted the less likely it is that adversaries are observing it. Although the belief may be expressed as the adversaries' success in compromising any number of relevant factors, such as relay software or physical location, ultimately it must describe the probability that the adversaries observe traffic on the virtual links into and out of the Tor network. A virtual link is an unordered pair of network hosts, and the entry virtual links consist of client-guard pairs while the exit virtual links consist of destination-exit pairs. An adversary is considered to observe a virtual link if it can observe traffic in at least one direction between the two hosts. Although the representation of an arbitrary distribution over all virtual links can be very large, Jaggard et al. [17] describe how distributions of likely Tor adversaries can be represented efficiently by aggregating host locations (e.g., at the AS level) and by identifying a small set of relevant compromise factors and indicating their dependencies in a Bayesian network.

To indicate how to trade off vulnerability to different adversaries, each user adopts a trust policy that pairs her trust belief with a weight for each adversary. Each weight is a number in $[0,1]$ that indicates the relative level of concern that the user has for the associated adversary. In this work, we assume that distinct adversaries do not collude. If a user were worried about two adversaries colluding, she could combine her beliefs about them into those for a single adversary.

Trust policies are quite general and can easily be used with many kinds of beliefs and sources of trust information. For example, previous work that considered each relay to have an independent and individual probability of compromise [21], [22] can be represented as a trust policy by including a single adversary and allowing him to compromise each relay independently with its given probability. As another example, previous work that considered as a potential threat each AS and IXP [13], [15], [24], [29] can be represented as a trust policy by including each AS and IXP as an adversary with equal weight and allowing each AS and IXP to compromise with probability 1 all virtual links passing through it. Moreover, as described by Jaggard et al. [17], trust policies can incorporate beliefs about a variety of other sources of network compromise, such as software vulnerabilities, physical cable tapping, and geographic location.

We do not expect that most individual users will craft their own trust policies. Indeed, doing so is likely best left to experts unless the user has strong and idiosyncratic beliefs or concerns. Rather, we envision that knowledgeable specialists, such as security researchers and professionals, will provide opinions about vulnerability to specific kinds of adversaries, and that institutions, such as governments and consumer advocacy groups, will incorporate these opinions into trust policies that are appropriate for their communities. An important special case of this is that we expect that the Tor Project would select a default policy that is in the broad interest of all Tor users, and then would configure the standard Tor client to use it as well as provide any necessary supporting data through the Tor network, much as Tor consensuses (i.e., hourly documents describing available relays) are distributed today by a set of directory authorities.

We will consider two specific trust policies in our analysis of TAPS: ( $i$ ) The Man, which models a single powerful global adversary whose exact location isn't known with certainty, and (ii) Countries, which models each country as an adversary whose locations are known exactly. Either of these policies constitutes a plausible default policy as well as that of a particular user community. We now describe these models.

\section{B. The Man}

The Man represents a powerful adversary who may create, compromise, or coerce the diverse entities that make up the Tor network. Specifically, we give The Man an independent probability to observe each Tor relay family, AS organization, and IXP organization. A relay self-identifies its family in a descriptor [40] that it uploads to the directory authorities, and an AS or IXP organization is identified using public information as being controlled by the same corporate or legal entity [10], [23]. Without any basis for differentiation, The Man compromises each AS and IXP organization independently with probability 0.1 . For relays, we consider that trust may increase the longer a given relay has been active. This will not guarantee protection against an adversary that is willing to contribute persistently-high levels of service to the Tor network. However, it can require adversaries to either make their own persistent commitments to the network or to have compromised others who have done so (and are thus most committed, experienced, and difficult to attack). For The Man, we therefore assume each family is compromised by the adversary independently with probability between 0.02 and 0.1 , where the probability increases as the family's longevity in Tor decreases. We calculate longevity as follows: First, relay uptimes are calculated as the exponentially-weighted moving average of the relay's presence in a consensus with Running, Fast, and Valid flags with a half-life of 30 days. A relay family's uptime is simply the sum of its relays' uptimes. The probability that a family is compromised is then taken as $(0.1-0.02) /($ family_uptime +1$)+0.02$.

\section{Countries}

As an alternative to The Man, the Countries trust policy includes as an adversary each individual country in the world. A particular country adversary compromises with probability 1 every AS or IXP that is located in that country and no others. All country adversaries are given a weight of 1 . This policy illustrates a geographic perspective for Tor security, and it also demonstrates how we handle multiple adversaries.

\section{A. Adversary Model}

\section{SECURITy Model AND Metrics}

As we have described in our trust model, we are considering an adversary who may control or observe some Tor relays and parts of the Internet infrastructure. Note that an important special case of this is that the adversary might observe the destination itself. From these positions, we then analyze the adversary's success in deanonymizing users via the following methods: $(i)$ performing a first-last correlation attack, (ii) identifying the relays used on an observed connection, and 
(iii) observing Tor connections over time and linking them as belonging to the same user.

As described earlier, first-last correlation attacks are possible whenever the adversary is in a position to observe traffic between the client and entry guard as well as between the destination and exit. In such a situation, we assume that the adversary can immediately determine that the observed traffic is part of the same Tor circuit and thereby link the client with its destination.

Even when the adversary is not in a position to perform a first-last correlation attack, he still may observe different parts of the circuit and use traffic correlation to link together those parts. In such a case, if the observed relays on the circuit are unusually likely for a particular client to haven chosen (e.g., because of atypical trust beliefs), then the adversary may be able to identify the client even without direct observation. This is even more of a concern if the adversary applies congestion or throughput attacks [14], [26] to indirectly identify the relays on a target circuit. Therefore, we will consider the ability of the adversary to identify the source and destination of an observed circuit based on knowledge of its relays.

Finally, it is important to consider multiple connections over time instead of just one in isolation. Every circuit that a client creates may give the adversary another opportunity obtain a sensitive position or may leak more information about the client. This problem is made worse by the fact that the adversary may be able to determine when some circuits are created by the same client. This could happen, for example, if the adversary repeatedly observes traffic to the destination and the client interacts with the same online service using a pseudonym or requests a sequence of hyperlinked documents. Observe that in both of these examples the linking is done using similarities in the content of traffic and not via any weakness in the Tor protocol. Thus we will consider an adversary who can link observed connections by client.

Note that we are not considering an adversary that can identify traffic content based only on the timing and volume of data, that is, an adversary that can perform website fingerprinting [41]. Also, in reality we can expect adaptive adversaries who continually learn and shift the allocations of their resources, but we only analyze static adversaries in this paper. However, adaptiveness can be captured to a certain extent already by defining trust policies with respect to adversary behavior over time. That is, the compromise probability for a relay or virtual link can represent the probability that it will at some point during a given period be observed by the adversary.

\section{B. Anonymity Metrics}

We evaluate anonymity using two kinds of metrics. The first kind will give empirical estimates of the speed and frequency of first-last correlation attacks. The second kind will provide worst-case estimates for the adversary's ability to identify the source or destination of streams that are only partially observed.

First-last correlation attacks are relatively simple and result in complete deanonymization, and therefore we are interested in accurate estimates for how likely they are to occur. Moreover, their success depends on the behavior of the user and of the circuit-creation algorithm, and therefore we can measure it empirically via simulation. Following Johnson et al. [23], we use the following as metrics: (i) The probability distribution of time until a client is deanonymized via a correlation attack; and (ii) The probability distribution of the fraction of streams that are deanonymized via a correlation attack.

Measuring the anonymity of multiple connections that are only partially observed is more difficult because it isn't clear how successful the adversary can be at both linking separate streams and indirectly identifying relays on a circuit. Therefore, we take a worst-case approach and consider the adversary's ability to guess the source (resp. destination) of a sequence of streams that have been linked as coming from the same client (resp. going to the same destination) and for which the circuit relays have been identified. We measure this ability as the posterior distribution over network locations. Note that we do not take into account the fact that the adversary knows that streams for which the client or destination is unknown can only travel over virtual links that the adversary does not observe. Ruling out certain virtual links is more challenging than just positively identifying traffic on a virtual link because it requires the false negative rate for traffic correlation to be extremely low (in addition to the existing requirement that the false positive be extremely low). Thus, we leave this extension to our analysis to future work.

\section{Trust-aware Path SElection}

\section{A. Overview}

We describe two variants of the Trust-Aware Path Selection algorithms (TAPS): (i) TrustAll, which is intended for systemwide deployment, and (ii) TrustOne, which works with Tor's existing path-selection algorithm and is intended for use by a minority of users. Two TAPS variants are needed to blend in with two different types of other users: those who do use trust in path selection and those who do not. The main approach of both algorithms is to choose guards and exits to avoid first-last correlation attacks while also blending in with other users. Parts of this approach are shared with some previouslyproposed path-selection security improvements [7], [13], [22]. However, TAPS includes several novel features that improve the security and performance issues of these proposals. We highlight those features before proceeding to describe the algorithms in detail.

First, TAPS uses an API that encapsulates flexible trust policies. These trust policies support adversaries from a very general class of probabilistic models. As previously described, this class can represent features such as uncertainty, multiple adversaries, and adversarial control of diverse network elements.

Second, TAPS clusters client locations and (separately) destination locations. Each location cluster has a representative, and all locations in the cluster are treated as if they were the representative. The main purpose of this clustering is to prevent leakage of location information over multiple connections that would occur if paths were selected differently for each pair of client and destination location. Treating all members of the cluster as if they were the representative maintains anonymity within the cluster. A secondary benefit is reducing the amount of information needed to represent trust policies by reducing the number of paths to and from guards and exits that need be considered.

Third, TAPS treats its set of entry guards collectively, that is, in a way that provides security when multiple circuits, potentially using different guards, are considered. TAPS chooses each additional guard in a way that minimizes the additional exposure of its entry paths. Moreover, once a set of entry 
guards is chosen, TAPS doesn't prefer one guard in the set over another when creating a connection to a given destination. This prevents the cross-circuit attack discussed in Sec. II. It also makes TAPS compatible with the Tor's current default configuration of one guard, as it does not depend on being able to choose the best guard among several for a given destination.

Fourth, TAPS provides a configurable tradeoff between security and performance by parameterizing how much relayselection deviates from ideal load-balancing. The Tor network is under heavy load relative to its capacity [5], and latency is dominated by queuing delay at the relays [18]. Thus good load balancing is essential to maintaining Tor's performance, which is itself a crucial factor in Tor's success.

\section{B. Trust API}

The TAPS algorithms work with the trust policies described in Sec. III via an Application Programming Interface (API). Jaggard et al. [17] describe how to represent such policies with a Bayesian network. However, such a representation may not be the most efficient for the computations needed during path selection. Therefore, we abstract those computations into an API, and we describe how they can be efficiently implemented for The Man and Countries policies in Sec. V-E. We assume that the API is implemented by the creator of the trust policy.

Several API functions take as an argument a network location. There are several possible levels of granularity at which a network location may be defined in TAPS, such as the Autonomous-System level or the BGP-prefix level. Using more fine-grained locations will result in more accurate predictions about the adversaries' locations and thus improve security, but it will also result in increased runtime for the TAPS algorithms (and likely for the API functions as well).

We assume that API users can provide a locations data structure that $(i)$ allows the locations to be enumerated, (ii) includes each location's popularity rank for Tor clients, (iii) allows IP addresses to be mapped to locations, and (iv) includes size of each location (e.g., the number of IP addresses originated by an Autonomous System).

We also assume that API users can provide a relays data structure that $(i)$ allows relays to be enumerated by unique identity keys (e.g., as represented by fingerprints [40]), (ii) includes the data in each relay's consensus entry (e.g., the status flags, weight, and IP address), and (iii) includes the data in each relay's descriptor (e.g., the exit policy).

The trust API functions are as follows:

1) LOCATIONDistance( $\left(l o c_{1}, l^{\prime} c_{2}\right.$, relays, weights): This function returns an abstract distance between two locations that measures the dissimilarity of the adversaries that appear on the network paths between the locations and the relays. This distance is the expected sum over relays weighted by weights of the total weight of adversaries that appear on one of the virtual links between the relays and $l o c_{1}$ and $l o c_{2}$ but not the other. This function turns the set of locations into a metric space.

2) GuARDSECURITy(client_loc, guards): This function returns a security score for the use of the given guards as entry guards by a client in location client_loc. The score must be in $[0,1]$, and it should represent the expected total weight of adversaries not present on the paths between client_loc and guards, normalized by the sum of all adversary weights. Thus a higher score indicates higher security.

3) EXITSECURITY(client_loc, dst_loc, guard, exit): This function returns a security score for the use of guard and exit by a client in location client_loc to connect to a destination in $d s t$ loc. The score must be a value in $[0,1]$, and it should represent the expected total weight of the adversaries that either are not present on the path between client_loc and guard or are not present on the path between dst_loc and exit (i.e., those not able to perform a correlation attack), normalized by the sum of all adversary weights. Thus, again, a higher score indicates higher security.

\section{TrustAll}

TrustAll consists of two separate processes:

1) Cluster: This process is run by the trust-policy provider (e.g., by the Tor directory authorities for the default policy). It clusters client and destination locations and makes the results available to clients. To maintain the anonymity sets provided by the clusters, this process should execute infrequently (e.g., every 6 months) and only to reflect significant changes in the trust on entry and exit virtual links. It takes the locations and relays data structures as inputs and produces clusters as output, which is a mapping from each location chosen as a cluster representative to the set of locations in its cluster.

2) Connect: This process is run by a Tor client. It runs every time a new connection is requested. It uses the output of the CLUSTER process, the state of the client (e.g., current network consensus, current circuits, and client IP address), locations, and relays. It may create a new circuit or reuse an existing one.

We now detail these processes.

1) Cluster: Network locations are clustered twice. One clustering will be applied to the destination's location during path selection, and the other will be applied to the client's location. The output of a clustering is a partition of network locations with a single member of each cluster in the partition designated as that cluster's representative. Client and destination clusterings are performed slightly differently because a single client is likely to visit many destinations. Therefore, if we were to bias destination clusters, we could potentially reduce security for all clients on at least one connection, but we can bias client clusters towards the most likely locations and improve security for most clients.

a) Clustering destination locations: Destinations are clustered with a k-medoids algorithm [32], modified to produce balanced-size clusters. Balance is needed for good anonymity, as each cluster is an anonymity set. The medoids of the resulting clusters are used as representatives. The destinationclustering algorithm takes two parameters: (i) num_clusters, the number of clusters to produce, and (ii) max_rounds, the maximum number of assignment rounds. The clustering is accomplished as follows:

1) Choose as an initial cluster representative a location uniformly at random from locations.

2) Choose the remaining num_clusters -1 cluster representatives by iteratively choosing the location with the largest distance to the representatives already chosen (i.e., the maximin distance), with distances determined by LOCATIONDISTANCE().

3) Assign locations to cluster representatives by greedily assigning to the smallest cluster (in terms of the total size of its locations) at a given time the location closest to its representative, as measured by LOCATIONDISTANCE(). 
4) Recalculate the cluster representatives by determining the location in each cluster with the smallest average distance to each other member in the same cluster.

5) Repeat from step (3) if any cluster representative changed and there have been fewer than max_rounds assignment rounds.

6) Return both the clusters and their representatives.

b) Clustering client locations: Client clustering uses known popular client locations as cluster representatives and then clusters the remaining locations in one round. The clientclustering algorithm takes as input num_clusters, the number of clusters to produce. It creates the set of cluster representatives by selecting the num_clusters most-popular client locations. The remaining locations are assigned to clusters by greedily assigning to the smallest cluster at any time as in the destination clustering.

2) Connect: The CONNECT process is invoked when a Tor client is requested to connect to a destination. We assume for now that any needed DNS resolution has been performed, and CONNECT has been given a destination IP address. Section V-F discusses how DNS resolution might occur.

The essential mechanism that TrustAll uses to improve security is to compute security scores for relays in the guard and exit positions and then to only select the highest-scoring relays for those positions. Guard security scores are determined with GUARDSECURITY(), which takes into account any existing guards when choosing a new one and thus provides security with respect to the entire guard set. Exit security scores are determined with EXITSECURITY(), which takes into account the guard to be used for the circuit and thus can mitigate firstlast correlation attacks.

Given scores for relays in position $p \in\{g, e\}$ ( $g$ for guard and $e$ for exit), the SECURERELAYS() function (Alg. 1) is used to determine the secure relays, that is, those relays with highenough scores to be selected for a given position. Note that in Alg. 1 ReverseSort $(X, Y)$ sorts the entries $x \in X$ by descending values $Y[x]$, and $\operatorname{LENGTH}(X)$ returns the number of entries in $X$. SECURERELAYs () identifies the highest score

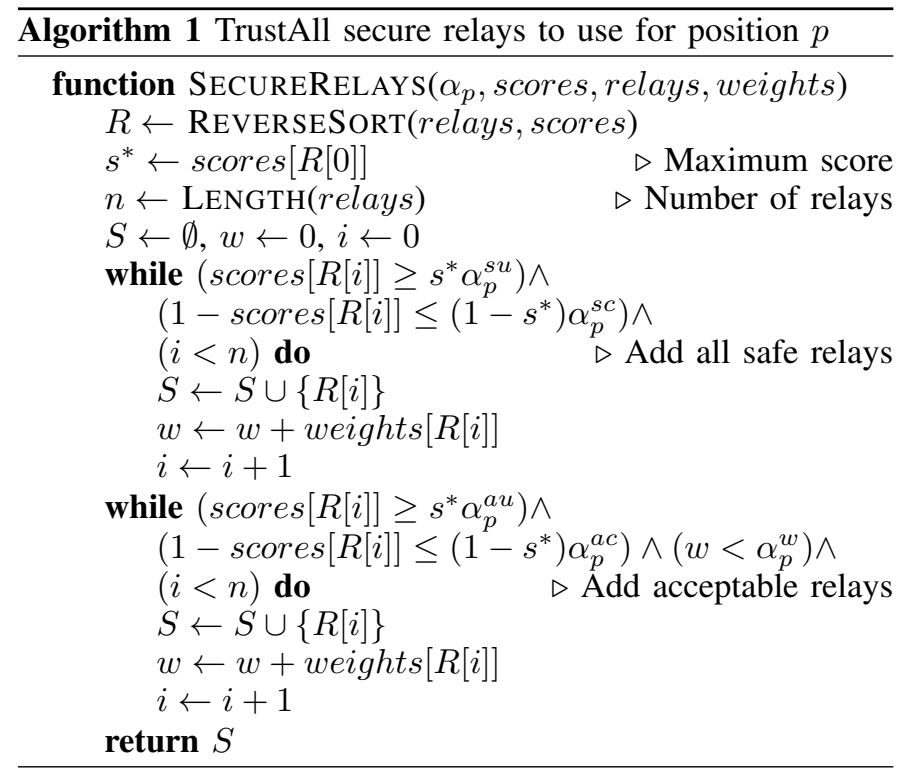

$s^{*}$. It adds to the set of secure relays all safe relays, that is, relays with scores very close to $s^{*}$. Then it considers the acceptable relays, that is, relays with scores close to $s^{*}$ but not close enough to make them safe. Acceptable relays are added in descending order of score until the desired fraction of the total bandwidth in position $p$ is reached. Distinguishing safe from acceptable relays enables improved load balancing when there are many highly-trusted choices available.

The parameters defining these sets are given as the list $\alpha_{p}=\left(\alpha_{p}^{s u}, \alpha_{p}^{s c}, \alpha_{p}^{a u}, \alpha_{p}^{a c}, \alpha_{p}^{w}\right) . \alpha_{p}^{s u}$ and $\alpha_{p}^{s c}$ are used just for safe relays, and $\alpha_{p}^{a u}$ and $\alpha_{p}^{a c}$ are used just for acceptable relays. Safe and acceptable relays are defined using the same method, but acceptable relays use less restrictive parameters, with $\alpha_{p}^{a u} \leq \alpha_{p}^{s u}$ and $\alpha_{p}^{a c} \geq \alpha_{p}^{s c}$.

The "uncompromised" parameter $\alpha^{u} \in\left\{\alpha_{p}^{s u}, \alpha_{p}^{a u}\right\}$ is used to include a relay only if it has a security score $s$ such that $s \geq$ $s^{*} \alpha^{u}$. It must be that $\alpha^{u} \leq 1$, or no relays would qualify. $\alpha^{u}$ is thus the required fraction of the maximum possible expected weight of adversaries with respect to whom the circuit position is considered uncompromised. One effect of this constraint is that relays with completely untrusted paths will not be chosen if there is at least one other option.

The "compromised" parameter $\alpha^{c} \in\left\{\alpha_{p}^{s c}, \alpha_{p}^{a c}\right\}$ is used to include a relay only if it has a score $s$ such that $1-s \leq$ $\left(1-s^{*}\right) \alpha^{c}$. It must be that $\alpha^{c} \geq 1$, or no relays would qualify. $\alpha^{c}$ is thus a limit on the multiple of the minimum possible expected weight of adversaries to whom the circuit position is considered compromised. An effect of this constraint is that if relays with completely trusted paths are available, then no other options are considered.

$\alpha_{p}^{w}$ represents the desired minimum bandwidth fraction of relays in position $p$ for the secure relays. It will be reached if the safe and acceptable relays together constitute at least that fraction. The weights argument to SECURERELAYS() maps relays to their positional bandwidth to determine if and when $\alpha_{p}^{w}$ is reached.

Let client_rep be the representative location for the client's cluster and $\overline{d s}$ t_rep be the representative location for destination's cluster. The CONNECT process proceeds as follows:

If the number $\ell$ of selected and responsive guards is less than the number $k$ desired (e.g., $k$ is the value of NumEntryGuards [40]), then $k-\ell$ new guards are selected. Each guard is added by $(i)$ creating scores where each potential guard $g$ has score GUARDSECURITY(client_rep, $G \cup\{g\}$ ), with $G$ is the current set of guards; (ii) identifying as the set of secure guards $S=$ SECURERELAYS $\left(\alpha_{g}\right.$, scores, $\left.P, g \_w e i g h t s\right)$, where $\alpha_{g}$ contains the guard security parameters, $P$ contains all potential guards not currently selected, and $g \_$weights contains the relays' weights for the guard position; and (iii) randomly selecting from $S$ with probability proportional to g_weights (i.e. making a bandwidth-weighted choice).

Consider the existing circuits in reverse order of the time a stream was most-recently attached. If current circuit $c$ is too dirty, that is, a stream was first attached too long ago (Tor's default dirtiness threshold is 10 minutes), then proceed to the next circuit. Otherwise, let $g_{c}$ be the circuit's guard, let $\alpha_{e}$ contain the security parameters for exit selection, let exits contain all potential exits for the desired connection according to the criteria Tor currently uses that don't depend on the guard (e.g., a compatible exit policy), and let $e_{-}$weights contain the relays' weights for the exit position. Let scores contain the exit scores, with scores $[e]=$ EXITSECURITY(client_rep, 
dst_rep, $\left.g_{c}, e\right)$ for all $e \in$ exits. Compute the set of secure exit relays $S=$ SECURERELAYS $\left(\alpha_{e}\right.$, scores, exits, e_weights). If the circuit's exit $e_{c}$ is in $S$, then reuse the circuit. Otherwise, proceed to the next circuit.

If no suitable circuit has been found and reused, let $c$ be the circuit among those that are not too dirty that most recently had a stream attached, and let $g_{c}$ be its guard. Choose a new exit $e$ by (i) creating scores where each $e \in$ exits has score EXITSECURITY (client_rep,dst_rep, $\left.g_{c}, e\right)$; (ii) identifying as the set of secure exits $\bar{S}=\operatorname{SECURERELAYS}\left(\alpha_{e}\right.$, scores, exits, $e_{-}$weights $)$; and (iii) randomly selecting from $S$ with probability proportional to e_weights. Reuse $c$ through its first two hops but "splice" $e$ onto the end after the middle relay. This effectively operates as a new circuit, but the handshakes through the first two hops are not repeated to reduce the latency of creating it.

If no circuit exists that is not too dirty, create a new circuit as follows: $(i)$ choose a guard $g$ uniformly at random from the $k$ selected and responsive guards, (ii) choose an exit $e$ as described for the case that a new exit is being spliced onto an existing circuit with $g$ as the guard, and (iii) choose a middle node as Tor currently does given $g$ and $e$ (e.g., bandwidth-weighted random selection). Note that, in contrast to vanilla Tor path selection, the guard and exit are not explicitly prevented from being contained in the same /16 subnet or relay family. Instead, the threat of entry and exit paths being observed by the same relay family or network is incorporated into the trust policy, and vulnerable paths are avoided by TAPS.

\section{TrustOne}

TrustOne path selection is designed to be used when most users are not using TAPS and instead are using vanilla Tor path selection. Thus slightly different behavior is required in order to fully blend in with the larger group. Also, if most users do not use trust, then more secure parameters can be used without impacting performance much.

As with TrustAll, TrustOne consists of a CLUSTER process and a CONNECT process. CLUSTER is performed in the same way as in TrustAll. The CONNECT process differs from that of TrustAll in the following ways:

- Securerelays() doesn't use the notions of safe and acceptable relays. It simply orders relays by their score and chooses the most secure up to the desired bandwidth fraction. The TrustOne version of this function appears in Alg. 2. Note that the performance parameter is a single value (i.e., $\alpha_{p}=\alpha_{p}^{w}$ ). TrustOne doesn't use the concept of acceptable relays because it must allow exit relays to be chosen the same they are in vanilla Tor path selection, which in TrustOne will happen when $\alpha_{e}^{w}=1$. Also, TrustOne can omit the distinction between safe and acceptable relays because load balancing is less important when few users are using trust.

- Given a guard, potential exits (i.e., the set over which scores are computed with ExITSECURITY()) are chosen exactly as they are in vanilla Tor path selection, including in particular the constraints preventing exits and guards from sharing a family or /16 subnet. This prevents a TrustOne user from being identified as using non-standard path selection (e.g., by a middle relay).

Note that a client can choose not to protect the fact the he is using TrustOne instead of vanilla Tor by setting a desired

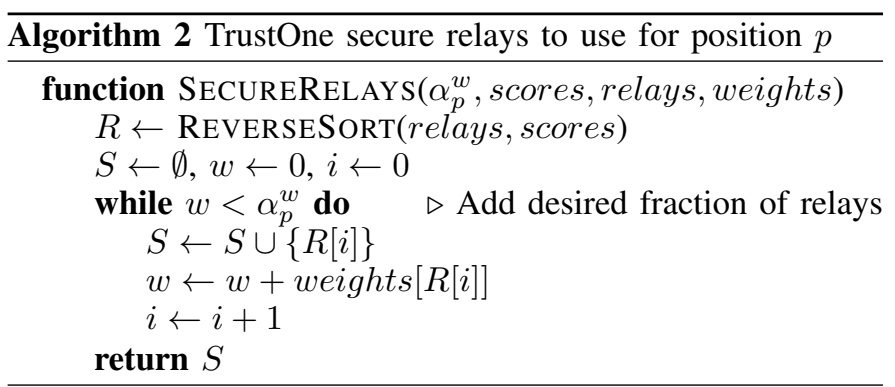

exit bandwidth fraction of $\alpha_{e}^{w}<1$. He may do this when he doesn't believe that revealing his use of TrustOne will reveal his identity, and using a smaller $\alpha_{e}^{w}$ will improve his security against a first-last correlation attack by restricting his circuits to more-secure exits.

\section{E. Trust API implementations}

The efficiency of the trust API depends on the type of trust policies used. For example, a user with trust in individual relays may only need to store a single trust value for each relay and perform simple arithmetic computations for the API functions, while a user with trust in Autonomous Systems may need to store an entire Internet topology and perform routing inference. In general, because the API functions return the expectation of values that can easily be computed if the compromised relays and virtual links are unknown, they can be implemented by repeatedly sampling the adversary distributions. Thus the API functions are compatible with the Bayesian-network representation of Jaggard et al. [17]. However, policies should use implementations that are efficient for their specific features.

For The Man policy, the trust API functions need access to data describing the relay families, AS organizations, IXP organizations, and the virtual entry and exit links on which each AS and IXP organization has a presence. The API functions can easily be implemented efficiently for The Man because there is a single adversary whose presence on a virtual link depends on the compromised status of network entities (i.e., relay families, AS organizations, and IXP organizations) that are each independently compromised. We implement the API functions as follows:

- LocationDistance $\left(l o c_{1}, l c_{2}\right.$, relays, weights $)$ : To compute this, consider each $r \in$ relays. Let $E_{1}$ be the set of network entities that exist between $r$ and both $l o c_{1}$ and $\operatorname{loc}_{2}$, let $E_{2}$ be the set of network entities that exist only between $r$ and $l o c_{1}$, and let $E_{3}$ be the set of network entities that exist only between $r$ and $l o c_{2}$. Let $p_{r}$ be the probability that the adversary is present on one of the paths from $r$ to $l o c_{1}$ and $l o c_{2}$ but not the other. $p_{r}$ is simply the probability that $(i)$ no $e \in E_{1}$ is compromised and (ii) either some $e \in E_{2}$ is compromised and no $e \in E_{3}$ is compromised or vice versa. The distance is computed as the weights-weighted sum of $p_{r}$ over $r \in$ relays.

- GuARDSECURITy(client_loc, guards): Let $E$ be the set of network entities between client_loc and the guards. The security score computed as the product of the probabilities that each $e \in E$ is individually uncompromised.

- ExitSecurity (client_loc,dst_loc, guard, exit): Let $E_{1}$ be the set of network entities that exist both between client_loc and guard and between dst_loc and exit, let 
$E_{2}$ be the set of network entities that exist only between client_loc and guard, and let $E_{3}$ be the set of network entities that exist only between $d s t_{-} l o c$ and exit. The security score is the product of the probability that no $e \in E_{1}$ is compromised and that either no $e \in E_{2}$ is compromised or no $e \in E_{3}$ is compromised.

For the Countries policy, the trust API functions need the list of all countries as well as a data structure mapping each relay to its country and each virtual link to the countries it passes through. LOCATIONDISTANCE $\left(l o c_{1}\right.$, $l o c_{2}$, relays, weights) is just a weighted sum over $r \in$ relays of the number of countries on which the virtual links $\left\{l o c_{1}, r\right\}$ and $\left\{l o c_{2}, r\right\}$ disagree. GUARDSECURITY(client_loc, guards) returns the fraction of countries not containing guards or on the virtual links between client_loc and guards. EXITSECURITY(client_loc,dst_loc, guard,exit) returns the fraction of countries either not containing guard and not on the $\{$ client_loc, guard $\}$ virtual link or not containing exit and not on the $\left\{d s t \_l o c, e x i t\right\}$ virtual link.

\section{F. Discussion}

So far, we have been discussing trust-aware routing to destination IP addresses. Many or most connections will require DNS resolution before the destination IP is known. Exit relays resolve DNS requests in current Tor routing to prevent linking of client IP address directly to a destination DNS request. This must also be done in a trust-aware manner, or there is little point in using trust-aware routing from exit to destination once the IP address is known. If we rely on a chosen exit to control the DNS resolution, then, even if it shares the default trust values, it may not be a good exit for resolving the intended destination. When doing iterative DNS resolution from the client, possibly switching to new circuits depending on the next identified DNS resolver, the performance overhead could be significant. In this paper, we assume that primary and backup nameserver ASes are included with exit-relay descriptors. Assuming that these are typically in the same AS as or immediately adjacent to the exit, this will at least make sure that initial DNS requests are trust-aware. How best to address DNS issues beyond that is outside the scope of this paper.

We expect the CLUSTER process to be performed by organizations (e.g., Tor or EFF) and the results distributed to users who trust their analysis and calculations. End-users would then only need to perform the CONNECT process. In the case of TrustAll, security depends on the assumption that many other users are using the same policy. Therefore, the TrustAll CONNECT process could be integrated with the standard Tor release and enabled with a default policy (e.g., The Man or Countries). However, TrustOne was designed to be used by a minority of users, and while the algorithm could be included with Tor, it would not be enabled by default. We analyze the security and performance of both approaches in the following sections.

\section{SECURITY ANALYSIS}

\section{A. Experimental Setup}

We experimentally evaluate the security of the TrustAll and TrustOne algorithms against The Man using an Internet map, data about the Tor network, and path-selection simulators for TAPS and for vanilla Tor. The AS-level routing map and past state of the Tor network are constructed as described in Sec. II, but for these experiments we use data from December 2013.
We augment the routing map using sibling information based on RIPE WHOIS records. We identify IXPs and place them on these on the AS-level paths using data from the IXP mapping project [8]. We group ASes into commercial organizations using the results of Cai et al. [10]. We conservatively group IXPs into organizations based on similarities in their listings in the Packet Clearing House and PeeringDB (see [23] for details).

We simulate path selection on past Tor networks using the Tor Path Simulator (TorPS) [23]. TorPS provides Monte Carlo simulation of user circuit creation over weeks and months on the changing Tor network. Each TorPS sample consists of a sequence of circuits and assignments to those circuits of requested user connections over the period of simulation. We use TorPS unmodified to evaluate the security of vanilla Tor, and we also modify TorPS to use the TAPS path selection algorithms.

We perform our TorPS simulations for two models of user behavior: the Typical model, and the IRC model. Johnson et al. describe these models in detail [23]. The Typical model consists of four 20-minute user traces obtained from actual (volunteer) user activity over Tor: (i) Gmail / Google Chat, (ii) Google Calendar / Docs, (iii) Facebook, and (iv) web search activity. It includes 205 unique destination IPs and uses TCP ports 80 and 443. These traces are played every day in five sessions between 9 a.m. and 6 p.m. This results in 2632 TCP connections per week. The IRC model uses the trace of a single IRC session to irc.oftc. net on port 6697, which we observe to resolve to 82.195 .75 .116 in AS 8365 (TU Darmstadt, DE). This model repeatedly plays the trace 8 a.m. to 5 p.m. every weekday. This results in 135 TCP connections per week.

To evaluate security with respect to The Man, we use it to draw a sample of the compromised relays and virtual links for each TorPS sample and consider the security of that sampled path-selection behavior against that sampled adversary. That is, we independently assign a compromised status to each AS organization, IXP organization, and relay family using the probabilities given in Section IV. We then consider the anonymity of the circuits in the TorPS sample against the sampled adversary. We run simulations over the first week of December 2013. We use 3 guards for all simulations.

\section{B. Location Clusters}

The TrustAll algorithm prevents the chosen Tor paths from revealing client and destination locations beyond their location clusters. An adversary that can identify the relays in each position of a circuit (e.g., by running a relay and being selected as a middle) may use them as evidence for the clusters of the client and destination. For example, if the adversary is also observing the exit-destination link, it may be the case that a given guard and exit would only be used to visit that destination by members of a given client cluster. As was shown in Section II, this is an especially powerful attack if the adversary can additionally link together multiple connections as belonging to the same (pseudonymous) user.

Thus we must consider the anonymity that is afforded when a client or destination is known to belong to a given cluster. In our experiments, we partition all Internet ASes into 200 clusters. This number of clusters allows for significant diversity in cluster behavior while reducing the anonymity set of roughly 3.7 billion addresses in IPv4 by a factor of 200 . We perform 


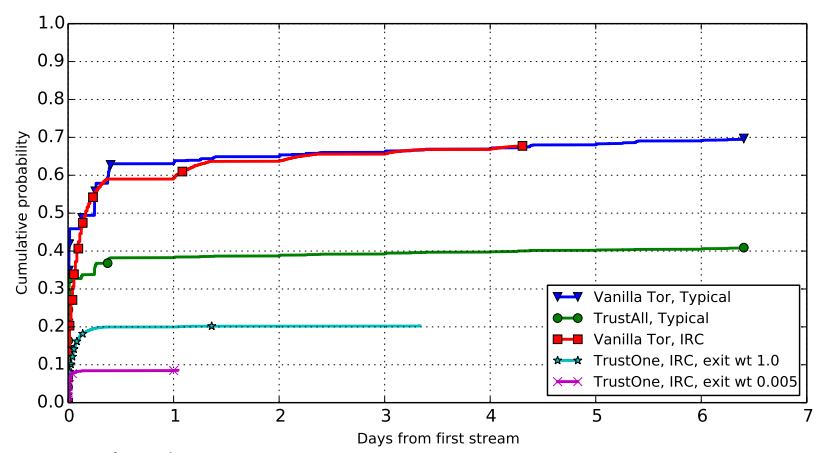

Fig. 4: Time to first compromise in The Man model

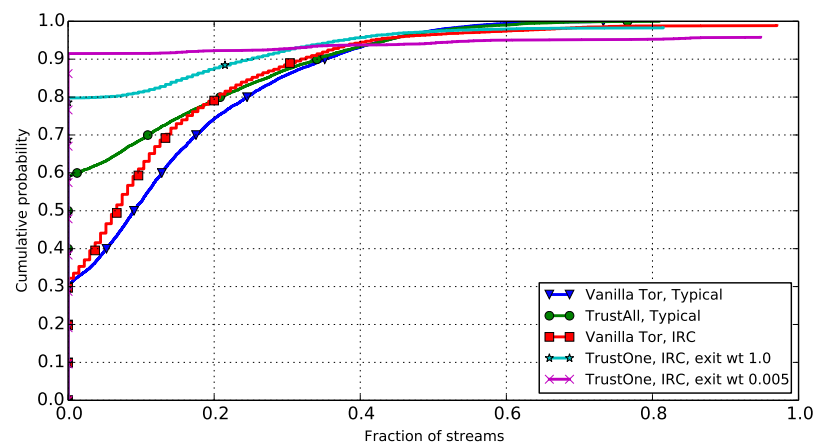

Fig. 5: Fraction of compromised streams in The Man model

clustering using as the guard and exit locations the sets of ASes in which Tor guards and exits were observed to reside during the six-month period from June 2013 to November 2013, which precedes the simulation period in December 2013.

Following the cluster-formation algorithm given in Section V, the 200 client clusters are created by choosing as cluster representatives the top 200 Tor client ASes reported by Juen [24]. In the resulting clustering, the median cluster size in terms of contained addresses is $11,363,072$, the minimum size is $10,840,321$, and the maximum size is $118,966,528$. Also as described in Section $\mathrm{V}$, the destinations clusters were formed slightly differently, using k-medoids clustering to identify representatives that were best able to minimize distances between cluster members and their representatives. The clusters that were the output of this process had a median of 11,992,641 IPv4 addresses, with a minimum of 11,358,466 and a maximum of $119,068,928$. Our clustering algorithm sought to maximize the number of addresses contained in each cluster, but it could easily incorporate other anonymity concerns, such as AS or country diversity.

\section{TrustAll Security}

First we consider security against The Man when all users use TAPS as the default path-selection algorithm (i.e., TrustAll). In particular we consider the threat of complete deanonymization via first-last correlation. We used the security parameters $\left(\alpha_{g}^{s u}, \alpha_{g}^{s c}, \alpha_{g}^{a u}, \alpha_{g}^{a c}\right)=(0.95,2.0,0.5,5.0)$, $\left(\alpha_{e}^{s u}, \alpha_{e}^{s c}, \alpha_{e}^{a u}, \alpha_{e}^{a c}\right)=(0.95,2.0,0.1,10.0)$, and $\left(\alpha_{g}^{w}, \alpha_{e}^{w}\right)=$ $(0.2,0.2)$. Figures 4 and 5 show cumulative distributions for when and how often deanonymization occurs for a Typical user in the most popular Tor client AS (6128) over 7 days of simulation.

We can see that TrustAll significantly reduces the chance of first-last correlation by The Man as compared to vanilla

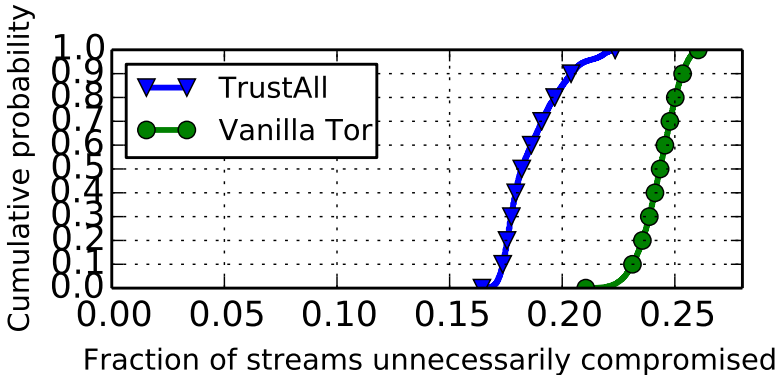

Fig. 6: Security in the Countries model

Tor. Users coming from AS 6128 see the probability of at least one successful first-last correlation attack drop from 0.7 to about 0.4. Observe that this overall reduction occurs both because the chance of choosing a compromised guard among the initial set of 3 is reduced (as seen in the values at 1 day) and because the chance of choosing additional compromised guards, precipitated by network churn, is reduced (as seen in the smaller slopes of the CDF). The results also show that the median fraction of compromised streams drops from around 0.1 to 0 .

Next we consider the security of TrustAll in the Countries model. In this model, users face multiple country adversaries (249), each of which deterministically compromises all ASes and IXPs within its borders. In this setting, users are sometimes necessarily compromised against those countries that contain both the source and destination AS. Thus we only consider the fraction of those streams that are to a destination AS in different country than the client AS and are "unnecessarily" compromised by some country. Figure 6 shows the distribution of this value for a Typical user in AS 6128 (which is in the US) active over seven days. It shows that TrustAll reduces the fraction of unnecessarily-compromised streams from a median of about 0.24 to a median of about 0.17 .

Finally, we consider security of IRC users using the TrustOne algorithm when default users are using vanilla Tor. In this case, the TAPS users choose guards and exits in a way dseigned to be sufficiently similar to how they are selected in vanilla Tor. Specifically, guards are selected using $\alpha_{g}^{w}=0.005$ and exits are selected using either $\alpha_{e}^{w}=0.005$ or $\alpha_{e}^{w}=1$. The former weight for exits results in a TrustOne user being 200 times more likely to have chosen a given exit than a vanilla Tor user. This could be an appropriate setting for a user who is not concerned with revealing his use of TrustOne and his trust beliefs. It could also be appropriate for a user who is just trying to protect anonymity for a single connection considered in isolation. The weight $\alpha_{e}^{w}=1$ results in exit selection by a TrustOne user that is identical to that of Tor users. This is an appropriate setting when the user wants to hide his use of TrustOne and the user's adversaries may be able to link circuits together over time as belonging to the same user.

Figures 4 and 5 shows the chance of deanonymization of an IRC user in AS 6128 via first-last correlation for TrustOne and vanilla Tor. We can see that TrustOne results in a significantly lower chance of compromise, from a 0.68 chance for vanilla Tor users to about 0.2 or 0.1 , depending on the exit-selection parameter $\alpha_{e}^{w}$. The median compromise rate also drops from about 0.7 to 0 . 


\section{PERformance AnAlysis}

The TAPS algorithm was designed to provide tunable performance while improving users' security by limiting the probability that an adversary can observe both sides of a Tor circuit. We now analyze the effect TAPS has on client performance and relay load balancing.

\section{A. Tor Network Model}

We evaluate the performance effects of TAPS using Shadow [3], [19], a scalable and deterministic discrete-event network simulator with a plug-in architecture that enables it to run real software. Shadow runs the Tor software, and so we can directly implement our algorithms in Tor's code base while increasing our confidence that the application-level performance effects are realistic.

We configure a private Tor deployment using Shadow and the large-scale topology produced by Jansen et al. [18]. Our base configuration consists of 400 Tor relays (including 4 directory authorities and 93 exits), 1380 Tor clients that also run a simple file-fetching application, and 500 simple file servers. Of the clients, 1080 are Web clients that are configured to: choose a random server and download a $320 \mathrm{KiB}$ file from it; pause for $[1,60]$ seconds chosen uniformly at random; and repeat. 120 of the remaining clients are bulk clients that are configured to repeatedly download a $5 \mathrm{MiB}$ file without pausing between downloads. Each experiment is configured to run for 1 virtual hour, which takes about 5 hours on our machine (using 12 Shadow worker threads) while consuming $40 \mathrm{GiB}$ of RAM.

We also run 180 ShadowPerf clients and model their behavior after the TorPerf [6] clients that measure and publish performance over time in the public Tor network. We compared ShadowPerf to TorPerf performance over several experiments and found that Shadow is able to model Tor performance characteristics with reasonable accuracy over a range of file download sizes.

\section{B. TAPS Implementation Details}

We branched Shadow [2] at commit 023055 eb5, shadowplugin-tor [4] at commit 9eed6a7c5, and Tor at version 0.2 .5.2-alpha and modified them to support experimentation with TAPS. The implementation of both the TrustOne and TrustAll versions of TAPS was done in 1126 lines of C code in Tor itself.

\section{TrustAll Against The Man}

Recall that in the TrustAll variation of TAPS, all users in the network are configured to select paths based on a common trust policy. We explore the performance of TAPS under different configurations of the parameters described in Section V. We use the same values of the parameters defining safe and acceptable relays in position $p \in\{g, e\}$ (i.e., $\alpha_{p}^{s u}, \alpha_{p}^{s c}$, $\alpha_{p}^{a u}$, and $\alpha_{p}^{a c}$ ) that were used for the security experiments in Section VI-C. We then adjust the required bandwidth fraction $\alpha^{w}=\alpha_{g}^{w}=\alpha_{e}^{w}$ in order to adjust the amount of load balancing that happens due to client path selection. Higher values of $\alpha^{w}$ relax security by requiring clients to consider more nodes in an attempt to exceed the bandwidth fraction and allow the algorithm to better distribute load among relays that have the capacity to support it (relays that are not safe or acceptable are never chosen in any case). Lower values of $\alpha^{w}$ reduce the number of relays that a client must consider, which means they effectively prefer more secure relays and perform less load balancing. We experiment with different values of $\alpha^{w}$ to explore these effects.

The results of our experiments are shown in Figure 7. Figure $7 \mathrm{a}$ shows the distribution of the time to receive the first byte of each download aggregated across all clients in our network. As can be seen, there is a significant and consistent improvement in latency to the first byte as $\alpha^{w}$ increases. As $\alpha^{w}$ increases, client load is better distributed because more clients will end up choosing high capacity nodes even if they are not the most secure choice. Similar results are shown for time to complete the downloads, for Web clients in Figure 7b and bulk clients in Figure 7c. Also, performance differences are consistent across the $\alpha^{w}$ settings for both Web and bulk clients, which we would expect because our path-selection algorithm is the same in both cases.

Our experiments resulted in decreasing performance as $\alpha^{w}$ decreases. We expect this to be the case since any deviation from Tor's default bandwidth-weighted algorithm will result in suboptimal load balancing. However, our results indicate that a clear performance-security trade-off is possible in TAPS and that the algorithm can be tuned to a desired level of performance while still removing the least secure relays from consideration.

A side effect of the decrease in performance is fewer completed downloads by each client over the course of the experiment due to our behavior models, as evident in figure $7 \mathrm{~d}$. Related to download times, there is a significant reduction in the number of downloads for clients (and a long neck for about 20 percent of Web clients). This is likely due to the fact that these clients, because of their location, consistently choose low capacity guards and exits that cause their downloads to receive bad performance. (Clients in the long neck of number of downloads are also in the long tail of download times.) This is also a result of our behavior models, in which clients do not start a new download until the previous one finishes. A richer behavior model in which some clients start multiple downloads at a time (e.g., representing users opening multiple tabs or starting multiple background file transfers) could alleviate this artifact.

As shown in Figure 7e, the reduction in the number of downloads also reduces total aggregate network throughput (bytes written summed across all relays every second). This again indicates a reduction in the ability of Tor to properly balance load when all clients in the network use TAPS. Again, $\alpha^{w}=1.0$ performs the closest to vanilla Tor and does not result in a significant loss in performance, despite removing the least secure relays during path selection.

Finally, Figure $7 \mathrm{f}$ shows the cumulative fraction of bandwidth weight from relays that fall outside of the safe thresholds but that were still considered during path selection. These relays represent those that were within the acceptable thresholds but not within the safe thresholds. Recall that TrustAll selects relays in this acceptable zone one at a time, from most to least secure, until the desired consensus weight fraction $\alpha^{w}$ is reached. As expected, the more performance that is demanded (i.e., as $\alpha^{w}$ increases), the more relays outside of the safe thresholds must be used to reach the desired performance. Our results indicate that there are settings of $\alpha^{w}$ that result in performance nearly as good as Tor's default performance-optimized algorithm, while also taking security into consideration. 


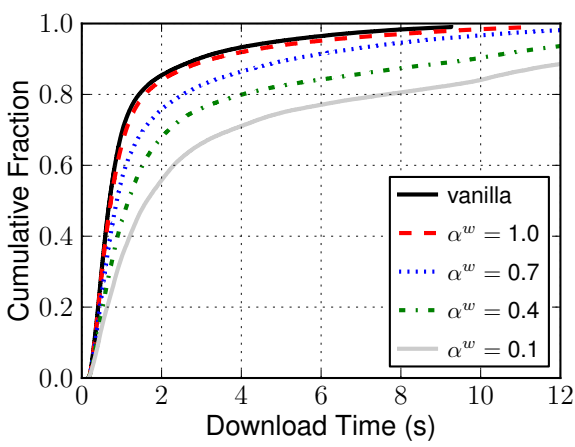

(a) Time to first byte of download per client

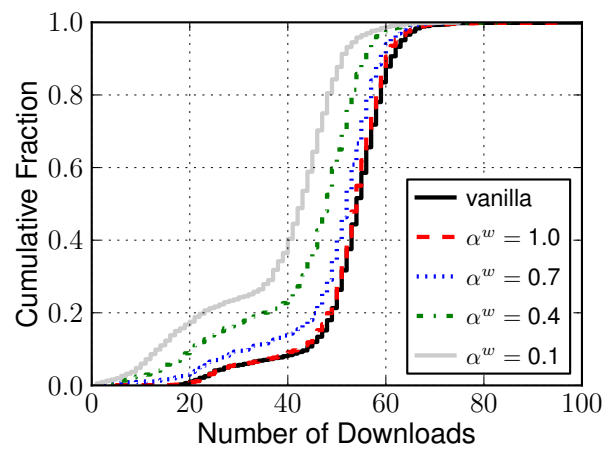

(d) Number of downloads per client
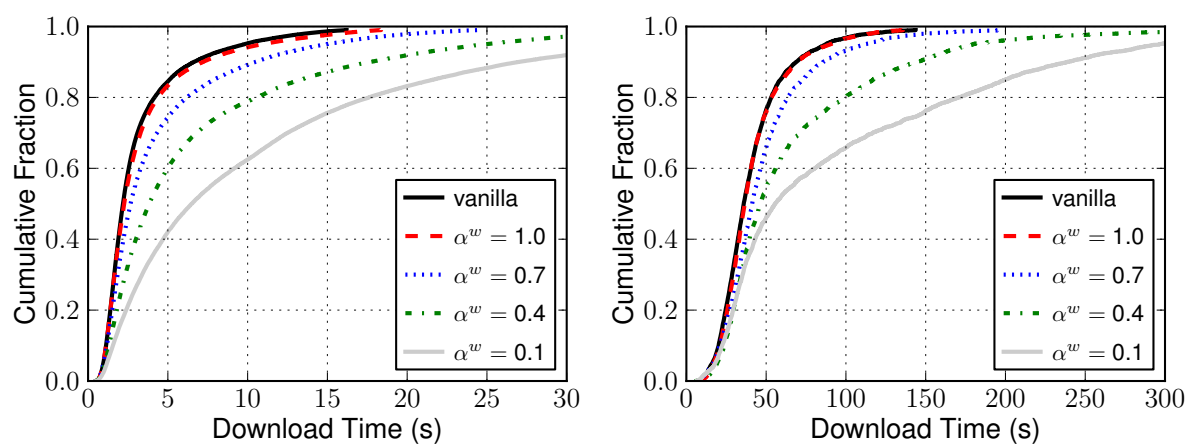

(b) Time to last byte of $320 \mathrm{KiB}$ download per client (c) Time to last byte of $5 \mathrm{MiB}$ download per client

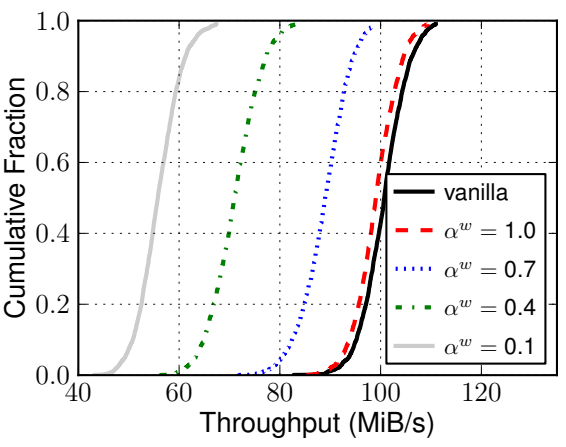

(e) Aggregate relay throughput per second

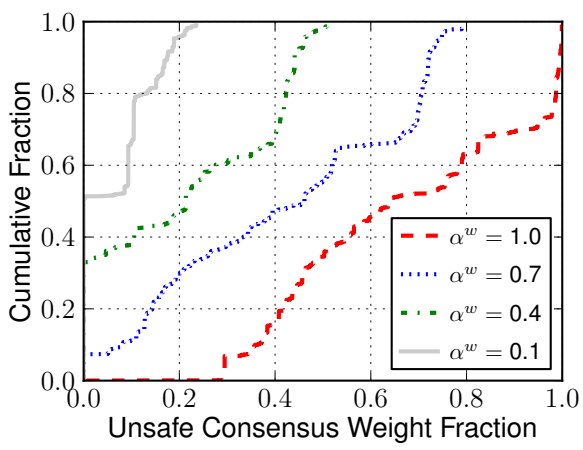

(f) Unsafe consensus weights considered

Fig. 7: Performance of the TrustAll variation of TAPS against The Man policy, varying required bandwidth fraction $\alpha^{w}$

\section{TrustAll Against Countries}

The experimental results discussed above were obtained using The Man policy. For completeness, we also experimented with the same parameters using the Countries policy. We confirmed that the same trends are present against the Countries policy as were discussed above, and the results increased our confidence in the conclusions drawn about the performance of TAPS. (The full set of graphs are excluded for space reasons.)

\section{E. Trading Security for Performance}

Figure 8 demonstrates how TAPS directly trades performance for security according to the parameter $\alpha^{w}$. Figure $8 \mathrm{a}$ shows the security-performance tradeoffs of TrustAll against The Man policy for various values of $\alpha^{w}$. Shown in the figure are two performance metrics: "Client Download Time" represents the median across all clients of the median download time for each client; "Relay Throughput" represents the median application throughput in terms of bytes written per second, accross all relays over all seconds during the experiments. Both of these metrics are normalized with respect to vanilla Tor, meaning that values closer to 1.0 indicates that TAPS achieves performance more similar to that achieved by vanilla Tor. Also shown in Figure 8a are the "Probability of Compromise" and the "Stream Compromise Rate" as metrics of security. The metrics are again normalized with respect to vanilla Tor, so that values closer to 0 are less similar to vanilla Tor and indicate higher security. As is clear in the figure, as the tradeoff parameter $\alpha^{w}$ increases, both of the performance metrics improve while both of the security metrics get worse. This is expected: as more relays are used to reach the performance requirements of $\alpha^{w}$, it is more likely that insecure relays or relays that exist on insecure paths will be selected and used in a circuit.

A similar analysis applies to the Countries policy, the results for which are shown in Figure 8b. The security metrics include the median fraction of "Unnecessarily Compromised Streams", where the source and destination of a stream do not exist in the same country and yet the stream was still compromised, and the median number of countries with which the client unnecessarily had a compromised circuit. The performance metrics are as above. The same basic trends hold for the Countries policy: as $\alpha^{w}$ increases and the number of potentially unsafe relays considered for a path increases, so does the number of avoidable stream compromises and the number of countries to which a given client is unnecessarily compromised. In all cases, however, security improves with respect to vanilla Tor while performance decreases depending on the tunable setting of the tradeoff parameter $\alpha^{w}$.

\section{F. TrustOne Against The Man}

In order for TrustAll to be effective, most clients must use it. If only a minority of clients use trust, then they should use TrustOne in order to blend in with vanilla-Tor users. They can also take advantage of their minority status by using highersecurity parameters without affecting Tor's load balancing much.

We demonstrate the performance of TrustOne by configuring 68 of our Web clients and 5 of our bulk clients to run the TrustOne algorithm with $\alpha_{g}^{w}=0.005$ and $\alpha_{e}^{w} \in\{0.005,1.0\}$; the other parameter settings are as in the TrustAll experiments. All other clients use the vanilla-Tor path-selection algorithm. 


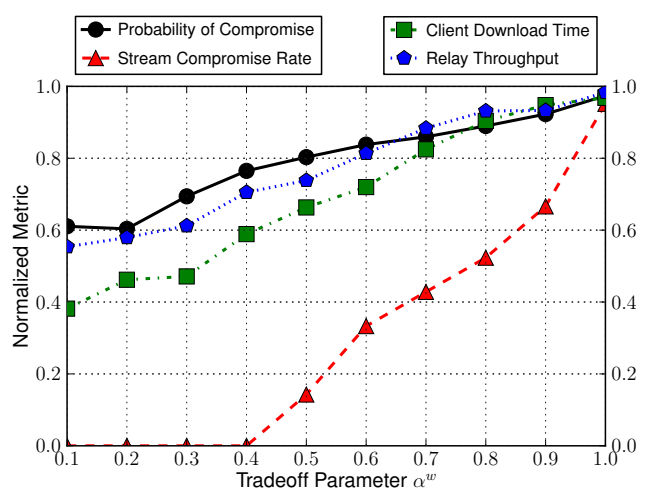

(a) The Man Policy

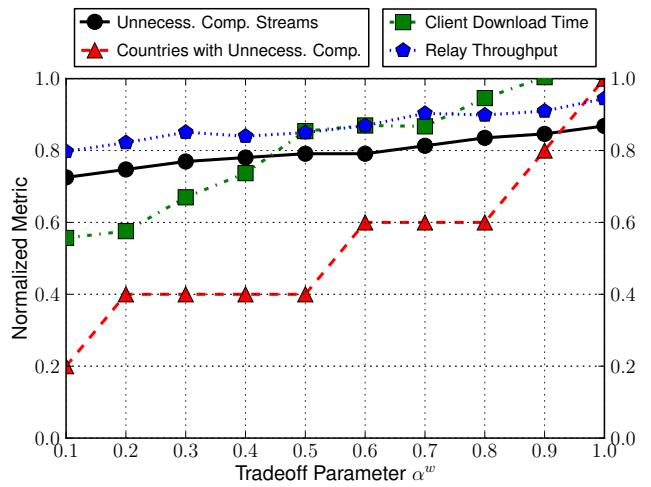

(b) Countries Policy

Fig. 8: Trading performance and security in TrustAll with $\alpha^{w}$

\begin{tabular}{|c||c|c|c|}
\hline metric & $\alpha^{w}=0.005$ & $\alpha^{w}=1.0$ & vanilla \\
\hline \hline Time to First Byte & $0.870,1.548$ & $0.783,1.694$ & $0.690,1.419$ \\
\hline Time to Last Byte 320KiB & $3.806,3.785$ & $2.685,3.255$ & $2.172,2.597$ \\
\hline Time to Last Byte 5MiB & $39.825,29.342$ & $35.203,14.395$ & $35.777,20.658$ \\
\hline Tor Throughput $(\mathrm{MiB} / \mathrm{s})$ & $98.635,4.893$ & $99.699,5.387$ & $100.660,4.250$ \\
\hline
\end{tabular}

TABLE II: Statistical summary (median, standard deviation) of performance for TrustOne

Thus the TrustOne clients choose secure guards, and they either choose exits identically to vanilla-Tor users in order to blend in $\left(\alpha_{e}^{w}=1.0\right)$ or don't attempt to hide their use of TrustOne and instead choose exits very securely $\left(\alpha_{e}^{w}=0.005\right)$.

Table II provides a statistical summary of the performance of the vanilla and trust clients. Note that the results for $\alpha^{w} \in\{0.005,1.0\}$ come from two separate TrustOne experiments, the vanilla-Tor results come from another experiment with no TrustOne clients, and the reported download times for $\alpha^{w} \in\{0.005,1.0\}$ are only for TrustOne clients. Across all three client performance metrics (time to first byte, and time to last byte of Web and bulk downloads), we see only a small drop in client performance for both settings tested. Although our sample size is small, both settings of $\alpha_{e}^{w}$ resulted in similar performance for the trusted user set. This indicates that performance for those clients was due to the capacity and congestion of their guard nodes (which they chose using a secure value of $\alpha_{g}^{w}$ ). Also shown in Table II are results showing that relay throughput in the TrustOne experiments was not significantly lower than in the vanilla Tor experiment (relay throughput is over all relays and thus in the TrustOne experiments includes traffic from both trust-aware and vanilla clients). This is attributable to a relatively small change in the load balancing across the network since only the trust users deviate from the optimized load balancing algorithm. Our results indicate that little performance is lost from using the TrustOne algorithm when a relatively small set of users are doing so.

\section{TRUST ERRORS}

Because the client's paths depend on her beliefs and may not be accurate, it is important to investigate the effects of errors in the client's beliefs on her security. Here, we do that experimentally by considering a variety of mismatches between client trust beliefs and the actual adversary. We look at three client behaviors against nine different actual adversaries for a single location (AS 6128) over one week. We also look at our Typical client in 401 different locations (the client ASes observed by Juen [24] and in our AS-level map for December 2013) with trust beliefs corresponding to The Man, but where the actual adversary distribution is one of a selected set of other behaviors.

The client might also have beliefs about the structure of the network. Errors in those may have significant impacts on the client's security if, for example, the client believes that an untrustworthy AS organization does not contain an AS that it actually does. We focus our experiments here on errors in trust beliefs, however.

We consider three different client behaviors: The Typical and IRC clients with The Man policy are as described above. We also consider a client with Typical network behavior who chooses paths based on trust beliefs that match the Countries adversary.

These client properties are combined with various adversaries, which may or may not match the client's beliefs and policies:

Type 0: The adversary is The Man adversary described above. Type 1: The probability of compromise is increased, relative to The Man, by a factor of 1.25 for AS/IXP organizations, lone ASes, and relay families. This reflects the possibility that the client uniformly underestimates the adversary's capabilities.

Type 2a: This is the same as The Man except ASes that are not part of any organization are not compromised.

Type 2b: This is the same as The Man except ASes that are not part of any organization are compromised with probability 0.05 .

Type 3: For each run, half of the AS organizations and half of the IXP organizations are compromised with probability 0.15 , and the others are compromised with probability 0.05. For efficiency, an AS that is not part of an AS organization is only assigned a compromised status when it is first encountered on a virtual link during analysis. Upon its initial observation, such an AS is assigned one of 0.15 and 0.05 as a compromise probability using a fair coin, and then it is compromised with that probability.

Type 4: The adversary is the same as The Man except longer uptime increases the compromise probability for a relay family (e.g., because of the increased chance of unpatched software). In particular, the compromise probability for a relay family with uptime $t_{f}$ is $0.1-(0.1-0.02) /\left(t_{f}+1\right)$.

Type 5: The adversary compromises each relay with probability 0.1 and each virtual link with probability $0.3439=1-0.9^{4}$. (The latter value is chosen to approximate the probability of compromising ASes/IXPs independently. On the virtual links that we consider, the median number of ASes/IXPs is four, 


\begin{tabular}{|l|c|c|c|c|c|c|c|c|c|}
\hline \multicolumn{10}{|c|}{ Client: Typical, against The Man } \\
\hline Adv. $\rightarrow$ & 0 & 1 & $2 \mathrm{a}$ & $2 \mathrm{~b}$ & 3 & 4 & 5 & 6 & 7 \\
\hline TTFC & $>7$ & $>7$ & $>7$ & $>7$ & $>7$ & $>7$ & .01 & $>7$ & $>7$ \\
\hline Prob. & .4 & .49 & .4 & .4 & .41 & .47 & .68 & .47 & .47 \\
\hline \hline \multicolumn{10}{|c|}{ Client: IRC, against The Man } \\
\hline Adv. $\rightarrow$ & 0 & 1 & $2 \mathrm{a}$ & $2 \mathrm{~b}$ & 3 & 4 & 5 & 6 & 7 \\
\hline TTFC & $>7$ & 4.17 & $>7$ & $>7$ & $>7$ & $>7$ & .07 & $>7$ & $>7$ \\
\hline Prob. & .41 & .5 & .41 & .41 & .41 & .48 & .71 & .49 & .48 \\
\hline \hline \multicolumn{8}{|c|}{ Client: Typical, against Countries } \\
\hline Adv. $\rightarrow$ & 0 & 1 & $2 \mathrm{a}$ & $2 \mathrm{~b}$ & 3 & 4 & 5 & 6 & 7 \\
\hline TTFC & .38 & .01 & .38 & .38 & .38 & .26 & .0 & .25 & .38 \\
\hline Prob. & .58 & .66 & .56 & .57 & .57 & .6 & .82 & .61 & .58 \\
\hline
\end{tabular}

TABLE III: Median time to first compromise (in days) and probability of compromise for three different client behaviors and nine different actual adversary distributions. Data are from 10,000 simulations of a client in AS 6128 running for 7 days.

\begin{tabular}{|l|c|c|c|c|c|c|c|c|c|}
\hline Adv. & \multicolumn{3}{|c|}{ Med. TTFC } & \multicolumn{3}{c|}{ Med. Prob. } & \multicolumn{3}{c|}{ Med. Frac. } \\
\hline & \multicolumn{3}{|c|}{ min./med./max. } & \multicolumn{3}{c|}{ min./med./max. } & \multicolumn{3}{c|}{ min./med./max. } \\
\hline 2a & .01 & $>1$ & $>1$ & .21 & .45 & .66 & .0 & .0 & .09 \\
\hline 4 & .01 & $>1$ & $>1$ & .27 & .49 & .69 & .0 & .0 & .11 \\
\hline 5 & .0 & .01 & .01 & .59 & .66 & .79 & .1 & .13 & .16 \\
\hline
\end{tabular}

TABLE IV: Statistics (minimum, median, and maximum) on the median times to first compromise (in days), compromise probability, and fraction of paths compromised over 10,000 trials for each of 401 client locations running for one day each against a selected set of adversaries. The clients choose paths against The Man; the actual adversary is shown in the first column.

although these are not necessarily from distinct organizations.) Type 6: The adversary is the same as The Man for ASes/IXPs. For relays and relay families, the adversary compromises nontrivial families with probability 0.1 and individual relays that are not part of a nontrivial family is 0.05 .

Type 7: The adversary is the same as The Man for ASes/IXPs. For relays and relay families, the adversary compromises families with probability $p_{\max }-\left(p_{\max }-p_{\min }\right) 2^{-\left(f_{s i z e}-1\right)}$, where $p_{\min }$ and $p_{\max }$ are the minimum (0.02) and maximum (0.1) probabilities of family compromise for The Man and $f_{\text {size }}$ is the number of relays in the family.

Table III shows the median time to first compromise (TTFC) in days and the probability that some circuit is compromised for the three different client types and nine different adversary distributions noted above. In each case, we take the client to be in AS 6128. The data are obtained from 10,000 simulations of client behavior from $12 / 1 / 13$ to $12 / 7 / 13$. Values of " $>7$ " for the TTFC indicate that the value is at least seven days, the length of these experiments.

Table IV shows various compromise statistics for a Typical client who chooses paths based on beliefs that match The Man against three different adversary distributions. For each of the 401 client locations, we ran 10,000 simulations and took the median TTFC, compromise probability, and fraction of compromised paths for that location. The table shows the minimum, median, and maximum of these per-location median values. Values of " $>1$ " for the TTFC indicate that the value is at least one day, the length of these experiments.

Comparing Table III with Fig. 4, we see that when the client in AS 6128 chooses paths against The Man, the use of TAPS increases her security, compared with vanilla Tor, against adversaries that are related to The Man even though the client is wrong about the exact nature of the adversary. More precisely, this is true for all of the adversary types considered in this section other than Type 5, which is the adversary that independently compromises each relay and virtual link and is the only type that does not compromise the network based on organizations and families. When the adversary is actually of Type 5, Tables III and IV show that it is able to do quite well against the client over many locations and client behaviors.

\section{Obtaining and Propagating Trust}

We consider as an example how much data must be stored and communicated to implement The Man policy. First, the client must be able to determine the cluster of itself and its destinations. With 46368 ASes in the network map used for TAPS analysis, 200 client clusters, and 200 destination clusters, $182 \mathrm{KiB}$ suffice for each client to determine the needed clusters. Second, to choose guards and exits, the client needs the ability to determine the AS and IXP organizations on any virtual link either between their cluster representative and a guard or between a destination-cluster representative and an exit. There are only 359 IXPs, and so an AS or IXP organizations can be specified in two bytes. For data gathered December 2013, all guards are within 603 ASes, all exits are within 962 ASes, and the average number of AS and IXP organizations on a virtual link is 4.05 . Thus a list of the entities on all the relevant virtual links for a given client is would be $1.68 \mathrm{MiB}$. Routing changes could be propagated daily or weekly with much smaller updates once the full data is obtained.

\section{Related Work}

An early proposals to use trust for Tor routing came from Øverlier and Syverson [31], who suggest choosing guards "based on trust in the node administrator". However, they do not develop this idea. A mathematical notion of trust in Tor was introduced by Johnson and Syverson [21]. They formalize trust as the probability of compromise of a relay, and they provide an analysis of end-to-end correlation attacks when there are just two different levels of trust. This model was later used by Johnson et al. [22] to produce a "downhill" Tor path-selection algorithm that can handle arbitrary trust levels at the relays and is designed to prevent traffic-correlation attacks. Jaggard et al. [17] greatly expand this probabilistic notion of trust by describing how to identify compromise factors that can apply to the links as well as the nodes, such as AS organizations, legal jurisdictions, and router software. They focus on expressing such rich trust models, while in this paper we focus on using these models in a path-selection algorithm that improves security.

Another approach to trust for anonymous communication is to explicitly leverage social network relations. Danezis et al. [12] describe this for interactive but low-volume anonymous communication. Concentrating on low-volume applications allowed them to make use of padding, which is generally too expensive and too ineffective for some of the more popular applications that use Tor. Mittal et al. [27] describe a socialnetwork onion-routing architecture designed for Web browsing and other interactive communications that adds resistance to an active adversary. This design uses potentially much longer paths than Tor's three hops to achieve intended security, and performance may suffer significantly as a result of this and other features of the design.

The threat of AS adversaries to Tor was first recognized by Feamster and Dingledine [15]. Their analysis shows that entry and exit paths through the network are likely to be simultaneously observed by a single AS $10 \%$ to $30 \%$ of the 
time, depending on the locations of the client and destination. They suggest that clients choose entry and exit nodes to avoid traversing the same AS upon entry and exit. Edman and Syverson [13] update this work and show that, despite the growth of the network from about 30 to about 1300 relays, the risk of denanonymization by a single AS is not reduced. They also show how to efficiently implement the AS-aware path selection suggested by Feamster and Dingledine by providing clients with routing data that enables them to infer AS-level routing paths. Murdoch and Zieliński [29] introduce IXPs as a potential adversary. They show that an IXP can correlate traffic even at low rates of sampling. Link adversaries at both ASes and IXPs were extended by Johnson et al. [23] to consider adversaries controlling multiple ASes or IXPs, such as companies that own many IXPs. Akhoondi et al. [7] present an alternate method for clients to efficiently infer the ASes between hosts for purposes of choosing Tor paths that avoid allowing the same AS to observe entry and exit traffic. Juen [24] presents another method for this purpose, this time with the addition of inferring IXPs on those paths. All of the preceding suggestions for AS-aware tor path selection neglect key details, such as how circuits are reused and how to handle destinations with no path that avoids putting an AS on both sides. In addition, Juen et al. [25] show that methods of AS inference for detecting Tor paths vulnerable to AS-level compromise suffer from significant false-positives and falsenegatives when compared to direct traceroute measurements.

Nithyanand et al. [30] present Astoria, which is the first reasonably-complete network-aware Tor path-selection algorithm. As described in Section II, like other previous work on network-aware path selection, Astoria is only secure when each connection is analyzed independently. DeNASA [9], by Barton and Wright, is another recent and fully-specified networkaware Tor path-selection algorithm. DeNASA only considers as adversaries individual ASes, and chooses to just protect against the eight ASes that are most likely to be in a position to deanonymize a connection. DeNASA also doesn't consider the specific destination when constructing a circuit, which allows it to use pre-built circuits for speed but makes it unable to protect connections to destinations with paths dissimilar from the pre-selected set used for exit selection. However, DeNASA is still vulnerable to leakage about a client's AS across repeated connections (assuming its guard and exit-selection algorithms are jointly used).

Sun et al. [36] show that traffic correlation attacks on Tor are effective even when the attacker observes paths in different directions on the entry and exit sides. They also demonstrate the application of BGP hijacking and interception attacks to redirect Tor traffic to malicious ASes in order to deanonymize users. Tan et al. [37] extend this analysis and show that $90 \%$ of Tor's bandwidth is vulnerable to BGP hijacking, and they propose as a defense a set of monitors to detect routing attacks and notify Tor clients to avoid the affected relays.

\section{CONCLUSION}

In this paper, we show how previous network-aware Tor path-selection algorithms are vulnerable to attacks across multiple Tor connections. We present TAPS, a path-selection algorithm for Tor that is not vulnerable to such attacks and that enables clients to avoid traffic-correlation attacks by using trust that they have in network elements. We present two globaladversary models, analyze the security and performance of
TAPS against these adversaries, and consider both trust errors and trust propagation.

Acknowledgments. We thank Ryan Wails for contributing to the results in Section II. The work at NRL was supported by ONR. Joan Feigenbaum's research was supported in part by NSF grants CNS-1407454 and CNS-1409599, DHS contract FA8750-16-2-0034, and William and Flora Hewlett Foundation grant 2016-3834.

\section{REFERENCES}

[1] "Alexa The Web Information Company. Top 1 million sites." https: //aws.amazon.com/alexa-top-sites/, retrieved August 26, 2016.

[2] "Shadow Git Repository," https://github.com/shadow/shadow.

[3] "Shadow Homepage," http://shadow.github.io/.

[4] "Shadow Tor plug-in Git Repository," https://github.com/shadow/ shadow-plugin-tor.

[5] “Tor Metrics Portal," http://metrics.torproject.org/.

[6] “TorPerf," https://gitweb.torproject.org/torperf.git/.

[7] M. Akhoondi, C. Yu, and H. V. Madhyastha, "LASTor: A low-latency AS-aware Tor client," in IEEE Symposium on Security \& Privacy, 2012.

[8] B. Augustin, B. Krishnamurthy, and W. Willinger, "IXPs: Mapped?" in Internet Measurement Conference, 2009.

[9] A. Barton and M. Wright, "DeNASA: Destination-naive as-awareness in anonymous communications," Proceedings on Privacy Enhancing Technologies, 2016.

[10] X. Cai, J. Heidemann, B. Krishnamurthy, and W. Willinger, "An organization-level view of the Internet and its implications (extended)," USC/ISI, Tech. Rep. ISI-TR-2009-679, 2012.

[11] "The CAIDA UCSD Internet Topology Data Kit - December 2013," http://www.caida.org/data/internet-topology-data-kit.

[12] G. Danezis, C. Diaz, C. Troncoso, and B. Laurie, "Drac: An architecture for anonymous low-volume communications," in Privacy Enhancing Technologies Symposium, 2010.

[13] M. Edman and P. Syverson, "AS-awareness in Tor path selection," in ACM Conference on Computer and Communications Security, 2009.

[14] N. S. Evans, R. Dingledine, and C. Grothoff, "A practical congestion attack on Tor using long paths," in USENIX Security Symposium, 2009.

[15] N. Feamster and R. Dingledine, "Location diversity in anonymity networks," in Workshop on Privacy in the Electronic Society, 2004.

[16] J. Geddes, R. Jansen, and N. Hopper, "How low can you go: Balancing performance with anonymity in Tor," in Privacy Enhancing Technologies Symposium, 2013.

[17] A. D. Jaggard, A. Johnson, S. Cortes, P. Syverson, and J. Feigenbaum, "20,000 in league under the sea: Anonymous communication, trust, MLATs, and undersea cables," Proceedings on Privacy Enhancing Technologies, 2015.

[18] R. Jansen, J. Geddes, C. Wacek, M. Sherr, and P. Syverson, "Never been KIST: Tor's congestion management blossoms with kernel-informed socket transport," in USENIX Security Symposium, 2014.

[19] R. Jansen and N. Hopper, "Shadow: Running Tor in a box for accurate and efficient experimentation," in Network \& Distributed System Security Symposium, 2012.

[20] A. Johnson, R. Jansen, A. D. Jaggard, J. Feigenbaum, and P. Syverson, "Avoiding the man on the wire: Improving tor's security with trustaware path selection," in To appear in Proceedings of the 24th Network and Distributed System Security Symposium (NDSS 2017). Internet Society, 2017.

[21] A. Johnson and P. Syverson, "More anonymous onion routing through trust," in IEEE Computer Security Foundations Symposium, 2009.

[22] A. Johnson, P. Syverson, R. Dingledine, and N. Mathewson, "Trustbased anonymous communication: Adversary models and routing algorithms," in ACM Conference on Computer and Communications Security, 2011.

[23] A. Johnson, C. Wacek, R. Jansen, M. Sherr, and P. Syverson, "Users get routed: Traffic correlation on Tor by realistic adversaries," in ACM Conference on Computer and Communications Security, 2013.

[24] J. Juen, "Protecting anonymity in the presence of autonomous system and internet exchange level adversaries," Master's thesis, University of Illinois at Urbana-Champaign, 2012.

[25] J. Juen, A. Johnson, A. Das, N. Borisov, and M. Caesar, "Defending tor from network adversaries: A case study of network path prediction," Proceedings on Privacy Enhancing Technologies, 2015. 
[26] P. Mittal, A. Khurshid, J. Juen, M. Caesar, and N. Borisov, "Stealthy traffic analysis of low-latency anonymous communication using throughput fingerprinting," in ACM Conference on Computer and Communications Security, 2011

[27] P. Mittal, M. Wright, and N. Borisov, "Pisces: Anonymous communication using social networks," in Network \& Distributed System Security Symposium, 2013.

[28] S. J. Murdoch and G. Danezis, "Low-cost traffic analysis of Tor," in IEEE Symposium on Security \& Privacy, 2005

[29] S. J. Murdoch and P. Zieliński, "Sampled traffic analysis by Internetexchange-level adversaries," in Privacy Enhancing Technologies Symposium, 2007.

[30] R. Nithyanand, O. Starov, P. Gill, A. Zair, and M. Schapira, "Measuring and mitigating AS-level adversaries against Tor," in Network \& Distributed System Security Symposium, 2016.

[31] L. Øverlier and P. Syverson, "Locating hidden servers," in IEEE Symposium on Security \& Privacy, 2006.

[32] H.-S. Park and C.-H. Jun, "A simple and fast algorithm for K-medoids clustering," Expert Systems with Applications, vol. 36, no. 2, 2009.

[33] J. Qiu and L. Gao, "AS path inference by exploiting known AS paths," in IEEE GLOBECOM, 2005.
[34] "Route Views Prefix-to-AS," http://data.caida.org/datasets/routing/ routeviews-prefix2as/, used data from 2015-10-01 08:00.

[35] "University of Oregon route views project," http://www.routeviews.org/.

[36] Y. Sun, A. Edmundson, L. Vanbever, O. Li, J. Rexford, M. Chiang, and P. Mittal, "RAPTOR: Routing attacks on privacy in Tor," in USENIX Security Symposium, 2015.

[37] H. Tan, M. Sherr, and W. Zhou, "Data-plane Defenses against Routing Attacks on Tor," Proceedings on Privacy Enhancing Technologies, 2016.

[38] “TC: A Tor control protocol (Version 1)," https://gitweb.torproject.org/ torspec.git/plain/control-spec.txt.

[39] "webfp/tor-browser-selenium: Tor Browser automation with Selenium.” https://github.com/webfp/tor-browser-selenium, fetched at commit 7ba36de.

[40] “Tor directory protocol," https://gitweb.torproject.org/torspec.git/blob/ HEAD:/dir-spec.txt.

[41] T. Wang and I. Goldberg, "Improved website fingerprinting on Tor," in Workshop on Privacy in the Electronic Society, 2013. 